\title{
Classification of braids which give rise to interchange
}

\author{
STEFAN FORCEY \\ FELITA HuMES
}

\begin{abstract}
It is well known that the existence of a braiding in a monoidal category $\mathcal{V}$ allows many higher structures to be built upon that foundation. These include a monoidal 2-category $\mathcal{V}$-Cat of enriched categories and functors over $\mathcal{V}$, a monoidal bicategory $\mathcal{V}$-Mod of enriched categories and modules, a category of operads in $\mathcal{V}$ and a 2-fold monoidal category structure on $\mathcal{V}$. These all rely on the braiding to provide the existence of an interchange morphism $\eta$ necessary for either their structure or its properties. We ask, given a braiding on $\mathcal{V}$, what non-equal structures of a given kind from this list exist which are based upon the braiding. For example, what non-equal monoidal structures are available on $\mathcal{V}$-Cat, or what non-equal operad structures are available which base their associative structure on the braiding in $\mathcal{V}$. The basic question is the same as asking what non-equal 2-fold monoidal structures exist on a given braided category. The main results are that the possible 2 -fold monoidal structures are classified by a particular set of four strand braids which we completely characterize, and that these 2 -fold monoidal categories are divided into two equivalence classes by the relation of 2 -fold monoidal equivalence.
\end{abstract}

57M99

\section{Introduction}

There are several levels of connection between the categorical concepts of braiding and interchange. The first study of these ideas was by Joyal and Street in [9]. They point out that a second tensor product in a given category which is a monoidal functor with respect to the first gives rise to a braiding, and vice-versa. Most recently the work of Balteanu, Fiedorowicz, Schwänzl, and Vogt in [3] includes description of the same correspondence in the context of lax monoidal functors. The connection between the $n$-fold monoidal categories in [3] and the theory of higher categories is through the periodic table as laid out in [2]. Here Baez and Dolan organize the $k$-tuply monoidal $n$-categories, by which terminology they refer to $(n+k)$-categories that are trivial below dimension $k$. The triviality of lower cells allows the higher ones to compose freely, and thus these special cases of $(n+k)$-categories are viewed as $n$-categories with $k$ multiplications. 
A $k$-tuply monoidal $n$-category is a special $k$-fold monoidal $n$-category. The specialization results from the definition(s) of $n$-category, all of which seem to include the axiom that the interchange transformation between two ways of composing four higher morphisms along two different lower dimensions is required to be an isomorphism. In [3] the $k$-fold monoidal categories have interchange transformations that are not isomorphisms. If those transformations are indeed isomorphisms then the $k$-fold monoidal 1-categories do reduce to the braided and symmetric 1-categories of the periodic table. Whether this continues for higher dimensions, yielding for example the sylleptic monoidal 2-categories of the periodic table as 3-fold monoidal 2-categories with interchange isomorphisms, is an open question.

The key requirement of a 2-fold monoidal structure on a category is that a second tensor product (in the sense of [9]) must be a functor which preserves the structure of the first tensor product. Technically we say that the second is a monoidal functor with respect to the first. When the two tensor products are identical, this translates into the existence of a coherent interchange transformation $\eta_{A B C D}:(A \otimes B) \otimes(C \otimes D) \rightarrow(A \otimes C) \otimes(B \otimes D)$. The chief goal of this paper is to study and classify the braids on four strands which can play the role of an interchange transformation in a braided category seen as a 2 -fold monoidal category. To be precise, given a braided category $(\mathcal{V}, \otimes, \alpha, c, I)$ (with strict units, a strong associator $\alpha$, and braiding $c$ ), we ask the central question: For which four-strand braids $b$ does the category $\mathcal{V}$ have in general a coherent 2-fold monoidal structure, when that structure has $\otimes_{1}=\otimes_{2}=\otimes$ as functors, has $\alpha^{1}=\alpha^{2}=\alpha$ as natural transformations, has strict unit $I$ for both identical tensor products, and has $x$ as the underlying braid of $\eta$ ?

For example, the standard choice of $\eta=1 \otimes c_{B C} \otimes 1$ (where $\otimes$ is associative) corresponds to the braid

$$
x=1 / 1 \text {. }
$$

There is a canonical epimorphism $\sigma: B_{n} \rightarrow S_{n}$ of the braid group on $n$ strands onto the permutation group. The permutation given by $\sigma$ is that given by the strands of the braid on the $n$ original positions. For instance on a standard generator of $B_{n}, \sigma_{i}$, we have $\sigma\left(\sigma_{i}\right)=(i \quad i+1)$. Candidates for interchange would seem to be those defined using any braid $x \in B_{4}$ such that $\sigma(x)=(23)$. However, it will be seen that many braids which at first seem to accomplish the same interchange (transposing the middle two terms and nothing else) do not in fact correspond to any $\eta$ which in general satisfies the axioms making $\mathcal{V}$ into a 2 -fold monoidal category. For contrast, here is a braid which turns out to exemplify this failure:

$$
x=|乡 y|
$$

Algebraic $8 \mathcal{G}$ Geometric Topology, Volume 7 (2007) 
Under the hypotheses in this central question, there are four more requirements on the braid $x$ which must be filled for the answer to be affirmative. We refer to them, in parallel to the axioms in [3], as internal and external unit conditions, and internal and external associativity conditions. This nomenclature is the same as for the corresponding axioms of a 2-fold monoidal category, which we will give in full in Section 3. We refer to the strands of a braid by their initial positions. A sub-braid will refer to the braid resulting from the deletion of a subset of the strands of a braid.

The internal unit condition on the four-strand braid $x$ is that the sub-braids resulting from deleting either the first two or the last two strands are both the identity (trivial braid) on two strands. The external unit condition is that the sub-braids resulting from deleting either the first and third strand or the second and fourth strand are again the identity on two strands.

Internal associativity is pictured as an equality in $B_{6}$ of two six-strand braids derived from the original four strand braid. We call the two derived six strand braids $L x$ and $R x$. $L x$ is algorithmically described as: (a) performing a copy of $x$ on the first 4 strands; (b) grouping the new first and second pairs as if the edges of two ribbons-the two pairs are actually $(1,3)$ and $(2,4)$; and (c) performing a copy of $x$ on the four new "strands"- the two ribbons along with the remaining two strands 5 and $6 . R x$ is described by three similar steps, but the initial copy of $x$ is on the last 4 strands, and the following ribbon edge pairing is on the pairs $(3,5)$ and $(4,6)$. The required equality, where $\sigma(x)=(23)$, is pictured here. Shading between two strands represents the ribbon edge pairing:

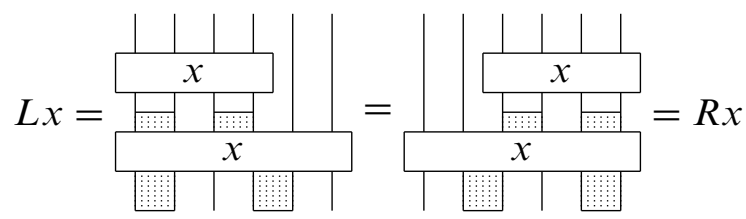

External associativity is pictured as an equality in $B_{6}$ of two six-strand braids derived from the original four strand braid. We call the two derived six strand braids $L^{\prime} x$ and $R^{\prime} x . L^{\prime} x$ is algorithmically described as: (a) pairing sets of strands $(2,3)$ and $(5,6)$ as if the edges of two ribbons; (b) performing a copy of $x$ on the four new "strands"the two ribbons along with the remaining two strands 1 and 4 ; and (c) performing a copy of $x$ on the new first four strands which are actually $(2,3,5,6) . R^{\prime} x$ is similarly described as (a) pairing sets of strands $(1,2)$ and $(4,5)$ as if the edges of two ribbons; (b) performing a copy of $x$ on the four new "strands"- the two ribbons along with the remaining two strands 3 and 6; and (c) performing a copy of $x$ on the new last four 
strands which are actually $(1,2,4,5)$. The required equality is

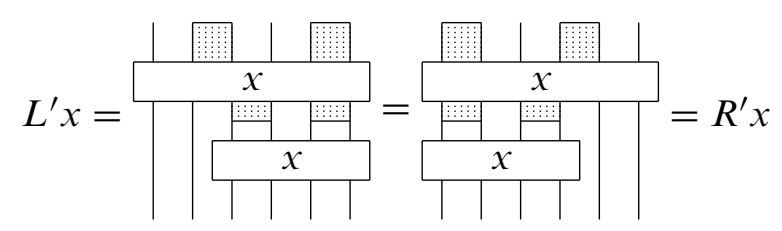

Example 2.9 and Example 2.11 contain pictures of original and derived braids. Notice that if $x$ is equal to its own 180 degree rotation, then $L^{\prime} x$ is the 180 degree rotation of $L x$, and $R^{\prime} x$ is the 180 degree rotation of $R x$.

The classification in Theorem 3.6 of four-strand braids which obey all four requirements turns out to be fairly simple. These braids, which can underlie a coherent interchange in a 2-fold monoidal category, we designate as (unital) interchanging braids. The first main result is that unital interchanging braids are precisely those given in terms of standard generators by

$$
b_{n^{ \pm}}=\left(\sigma_{2} \sigma_{1} \sigma_{3} \sigma_{2}\right)^{ \pm n} \sigma_{2}^{ \pm 1}\left(\sigma_{1} \sigma_{3}\right)^{\mp n}
$$

for $n$ a non-negative integer. For example $b_{2}+$ appears as

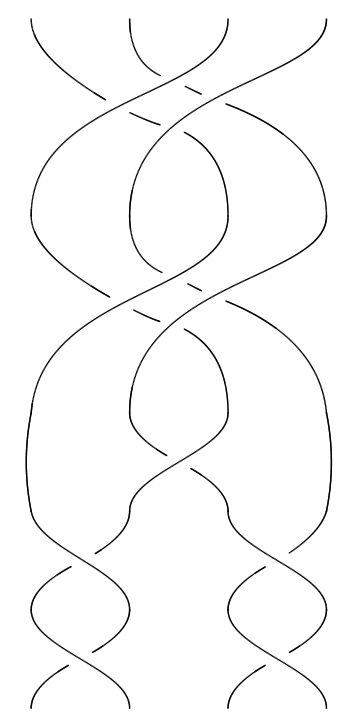

Several geometrical facts can be observed about these unital interchanging braids. First, the braid $b_{n^{ \pm}}$is a special element from the double coset $H \sigma_{2}^{ \pm 1} K$ of the braid group on four strands. Here $H$ is the subgroup generated by the braid $\sigma_{2} \sigma_{1} \sigma_{3} \sigma_{2}$ and $K$ is the subgroup generated by $\sigma_{1} \sigma_{3}$. Second, the braids $b_{n^{ \pm}}$are each equal to their own 
180 degree rotations. For example we have

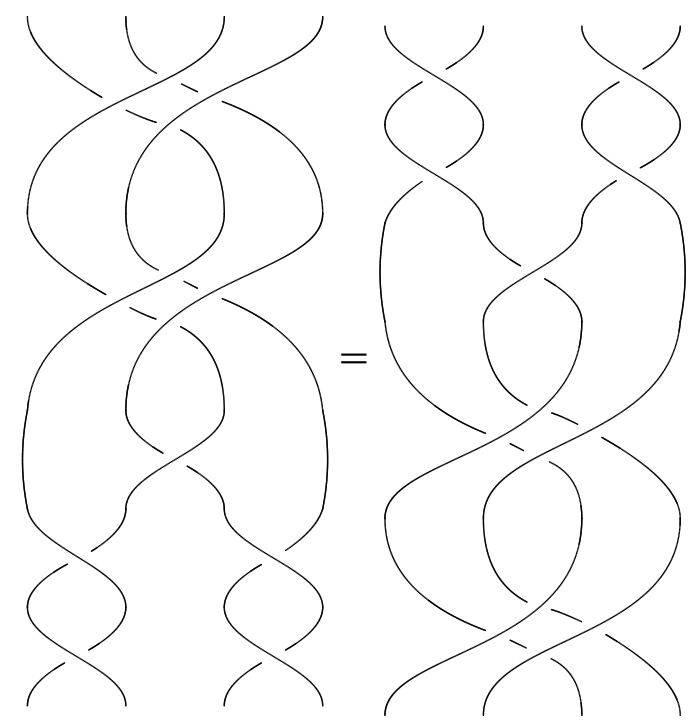

The best way to visualize this equality is to draw a rectangle around the "center" portion of the left braid, where a single copy of $\sigma_{2}$ divides the two "double stranded positive crossings" above from the two "negative crossings in tandem" below. Now imagine rotating this rectangle out of the plane of the page so as to uncross the upper double stranded crossings. After one full rotation (in order to completely undo the double stranded crossings) the right hand braid is achieved. A good exercise would involve asking that this geometric argument be made into an inductive proof. The braid equality in terms of generators is

$$
b_{n^{ \pm}}=\left(\sigma_{2} \sigma_{1} \sigma_{3} \sigma_{2}\right)^{ \pm n} \sigma_{2}^{ \pm 1}\left(\sigma_{1} \sigma_{3}\right)^{\mp n}=\left(\sigma_{1} \sigma_{3}\right)^{\mp n} \sigma_{2}^{ \pm 1}\left(\sigma_{2} \sigma_{1} \sigma_{3} \sigma_{2}\right)^{ \pm n} .
$$

Thus the third resulting fact is that the braid $b_{n^{ \pm}}$is in the intersection $H \sigma_{2}^{ \pm 1} K \cap$ $K \sigma_{2}^{ \pm 1} H$. It would be interesting to know whether or not the only braids in this intersection are the braids $b_{n^{ \pm}}$. It would also be interesting to know what connection, if any, there is to the the similar braid equalities which arise in the theory of tortile categories, as in Shum [14].

The rest of this paper proceeds as follows: In Section 2 we begin with a review of the category $\mathcal{V}$-Cat of enriched categories over a braided category $\mathcal{V}$. This is due to the fact that when $\mathcal{V}$ is braided then $\mathcal{V}$-Cat can be equipped with a monoidal structure. It turns out that the central question of which braids can underlie a coherent interchange is equivalent to the question of which braids can underlie the middle four interchange of the composition morphisms for a tensor product on $\mathcal{V}$-Cat. To be precise, given a braided category $(\mathcal{V}, \otimes, \alpha, c, I)$ (with strict unit, a strong associator $\alpha$, and braiding $c$ ), 
we ask the new question: For which four-strand braids $b$ does the 2 -category $\mathcal{V}$-Cat have in general a coherent monoidal structure, given canonical choices for the objects, hom-objects, and unit morphisms of the tensor product of two enriched categories, and the canonical choice for the associator in $\mathcal{V}$-Cat, and given $b$ as the underlying braid of the middle four interchange $\eta$ ?

In Forcey [6] it is shown that the external and internal unit conditions of a 2-fold monoidal category $\mathcal{V}$ imply the unital nature of $\mathcal{V}-$ Cat and the unit axioms for a product of $\mathcal{V}$-categories respectively. The external and internal associativity conditions imply respectively the $\mathcal{V}$-functoriality of the associator in $\mathcal{V}$-Cat and the associativity of the composition morphisms for tensor products of $\mathcal{V}$-categories. Here we actually move in the opposite direction of implication: in order to find evidence of sufficiency of the conditions which a braid must meet to be interchanging we find tensor products on $\mathcal{V}$-Cat which have the interchanging braids underlying their middle four interchange.

In Section 3 we review the axioms of a 2 -fold monoidal category and demonstrate the necessity of the conditions for our main result. In Section 4 we ask which of the 2-fold monoidal categories we have described as arising from a certain braided category are equivalent as 2 -fold monoidal categories. Our result is that the relation of equivalence of 2 -fold monoidal categories splits our interchanging braids into into two equivalence classes, represented by $\sigma_{2}$ and $\sigma_{2}^{-1}$. Section 5 gives a list of easily detectable obstructions which prevent a braid from having the interchanging property that is, which prevent it from being equivalent to one of the braids described by our main result. The value of our classification is principally to provide a solid framework for proofs about structures based upon a braiding. It turns out that only certain braids can correspond to interchange transformations. Thus our results can be used to provide cases for proofs, either by treating all the cases up to braid equivalence or more often just by treating representative cases of categorical equivalence classes.

As an example in Section 6 we generalize results mentioned by Joyal and Street. They point out that the category of enriched categories over a braided category is in general not braided and that taking the opposite is not an involution. We give a proof which uses knot theory to demonstrate non-existence in general for all possible interchanges. In questions of classification of structures in a specific monoidal category, our result on interchanges may be necessary in order to construct a complete picture. In Section 7 we relate our results to classification of operads in a braided category. Throughout we work in monoidal categories with a coherent strong associator, where "strong" implies that the natural transformation in question is an isomorphism. The units will however be strict.

Algebraic $8 \mathcal{G}$ Geometric Topology, Volume 7 (2007) 
Thanks are due to many whose time was contributed to the development of this paper. Included are the referees, who offered excellent suggestions for improvement of clarity, as well as Imre Tuba, Steve Lack, Jesse Siehler and Ross Street.

\section{Braiding and enrichment}

First we briefly review the definition of a category enriched over a monoidal category $\mathcal{V}$. Enriched functors and enriched natural transformations make the collection of enriched categories into a 2-category $\mathcal{V}$-Cat. The definitions and proofs can be found in more or less detail in Kelly [10], Eilenberg and Kelly [5] and of course in Mac Lane [11]. Some are included here for easy reference.

2.1 Definition A monoidal category is a category $\mathcal{V}$ together with a functor $\otimes: \mathcal{V} \times$ $\mathcal{V} \rightarrow \mathcal{V}$ and an object $I$ such that

(1) $\otimes$ is associative up to the coherent natural isomorphisms

$$
\alpha_{A B C}:(A \otimes B) \otimes C \rightarrow A \otimes(B \otimes C)
$$

called associators. The coherence axiom is given by the usual commuting pentagonal diagram as in [11].

(2) In this paper, $I$ is a strict $2-$ sided unit for $\otimes$.

2.2 Definition A (small) $\mathcal{V}$-Category $\mathcal{A}$ is a set $|\mathcal{A}|$ of objects, a hom-object $\mathcal{A}(A, B) \in|\mathcal{V}|$ for each pair of objects of $\mathcal{A}$, a family of composition morphisms $M_{A B C}: \mathcal{A}(B, C) \otimes \mathcal{A}(A, B) \rightarrow \mathcal{A}(A, C)$ for each triple of objects, and an identity element $j_{A}: I \rightarrow \mathcal{A}(A, A)$ for each object. The composition morphisms are subject to the associativity axiom which states that the following pentagon commutes:

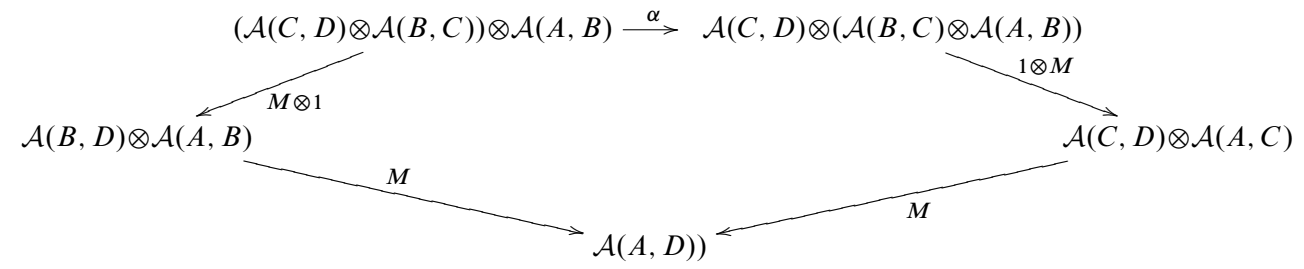

Algebraic $8 \mathcal{G}$ Geometric Topology, Volume 7 (2007) 
and to the unit axioms which state that both the triangles in the following diagram commute

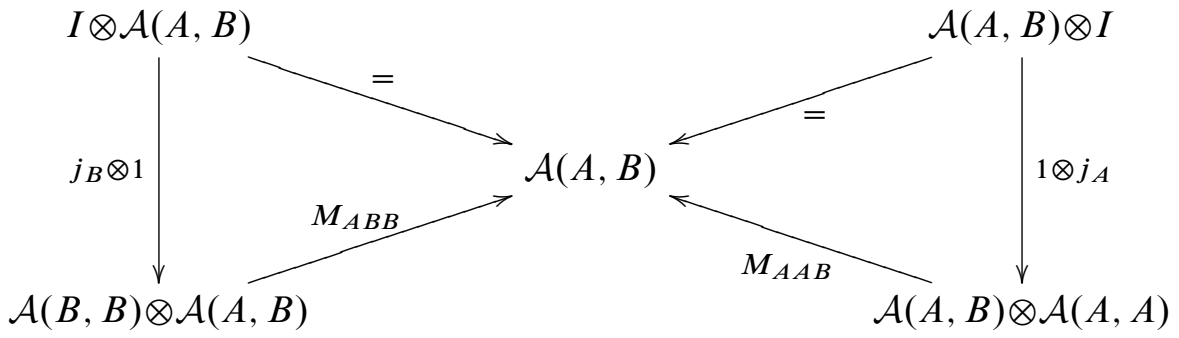

If $\mathcal{V}=$ Set then these diagrams are the usual category axioms. Basically, composition of morphisms is replaced by tensoring and the resulting diagrams are required to commute. The next two definitions exhibit this principle and are important since they give us the setting in which to construct a category of $\mathcal{V}$-categories.

2.3 Definition For $\mathcal{V}$-categories $\mathcal{A}$ and $\mathcal{B}$, a $\mathcal{V}$-functor $T: \mathcal{A} \rightarrow \mathcal{B}$ is a function $T:|\mathcal{A}| \rightarrow|\mathcal{B}|$ and a family of morphisms $T_{A B}: \mathcal{A}(A, B) \rightarrow \mathcal{B}(T A, T B)$ in $\mathcal{V}$ indexed by pairs $A, B \in|\mathcal{A}|$. The usual rules for a functor that state $T(f \circ g)=T f \circ T g$ and $T 1_{A}=1_{T A}$ become in the enriched setting, respectively, the commuting diagrams

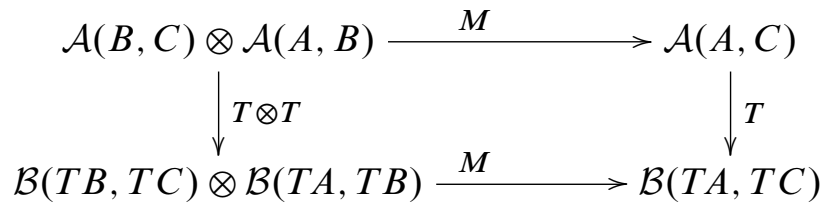

and

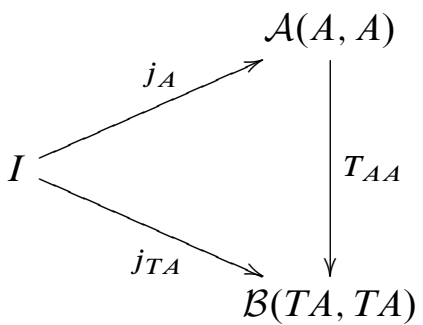

$\mathcal{V}$-functors can be composed to form a category called $\mathcal{V}$-Cat. This category is actually enriched over Cat, the category of (small) categories with Cartesian product.

2.4 Definition A braiding for a monoidal category $\mathcal{V}$ is a family of natural isomorphisms $c_{X Y}: X \otimes Y \rightarrow Y \otimes X$ such that the following diagrams commute. They are drawn next to their underlying braids. Recall that by "underlying braid" of a composite 
of braidings and associators between two products of the same $n$ objects, we refer to the $n$-strand braid with crossings corresponding precisely to each instance of the braiding and its inverse.

(1)
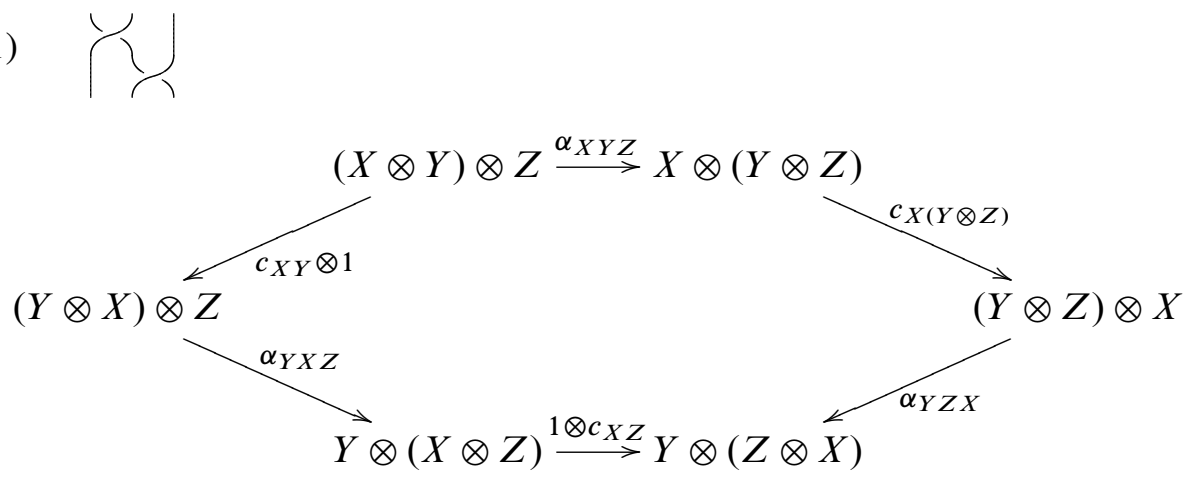

(2)
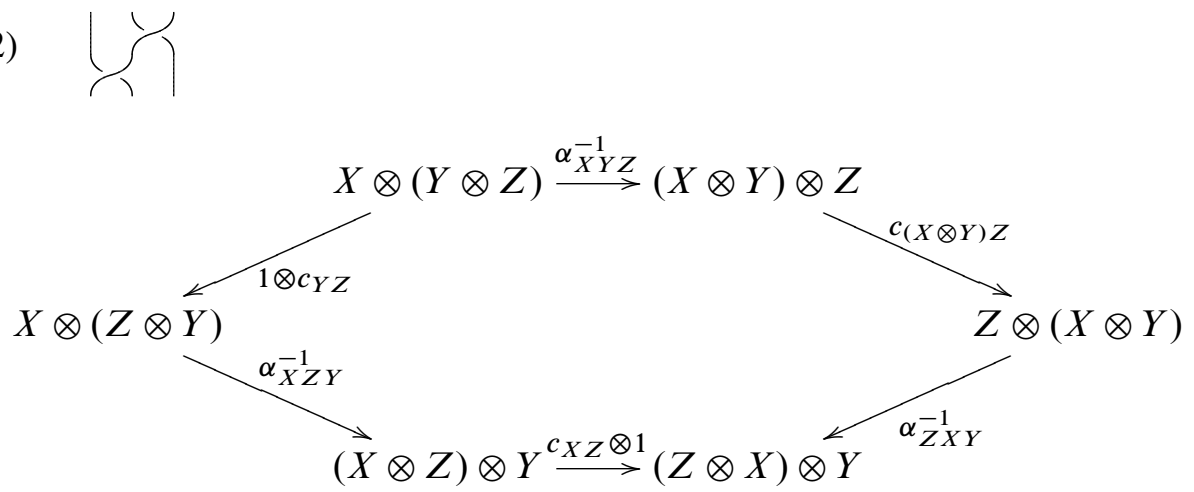

A braided category is a monoidal category with a chosen braiding. We will assume a strict unit in the monoidal categories considered here which implies a strict respect for units by the braiding. That is, $c_{I A}=c_{A I}=1_{A}$.

Joyal and Street proved the coherence theorem for braided categories in [9], an immediate corollary of which is that in a free braided category generated by a set of objects, a diagram commutes in general if and only if all legs having the same source and target have the same underlying braid.

2.5 Definition A symmetry is a braiding such that the following diagram commutes

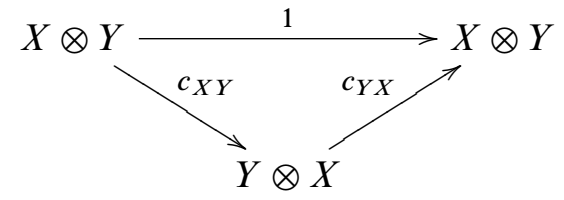

Algebraic 83 Geometric Topology, Volume 7 (2007) 
In other words $c_{X Y}^{-1}=c_{Y X}$. A symmetric category is a monoidal category with a chosen symmetry.

As pointed out by Joyal and Street, it is true that $c^{-1}$ is a braiding whenever $c$ is. These two braidings are equivalent if and only if $c$ is a symmetry; see Theorem 4.2 for the proof of this. It should be noted that there is immediately an obstruction to other potential braidings based on the original. For sake of efficiency we use notation $c_{A B}^{n}=c_{A B} \circ c_{B A} \circ c_{A B} \circ \cdots \circ c_{A B}$ where there are $n$ instances of $c$. It appears at first that if $c_{A B}$ is a braiding then $c^{\prime}=c^{ \pm(2 n+1)}$ is potentially a braiding for any $n$, but actually we find that:

2.6 Lemma for $n \geq 1, c^{\prime}=c^{ \pm(2 n+1)}$ is a braiding if and only if $c$ is a symmetry. (In that case $c^{ \pm(2 n+1)}$ is also a symmetry.)

Proof The obstruction arises from the the braided coherence theorem applied to the hexagonal diagrams with $c^{ \pm(2 n+1)}$ in place of the original instances of $c$. Observe that when we test the potential braiding for $n=1$, the hexagonal diagram (1) of Definition 2.5 has legs with the following two underlying braids. As denoted, this is an inequality:

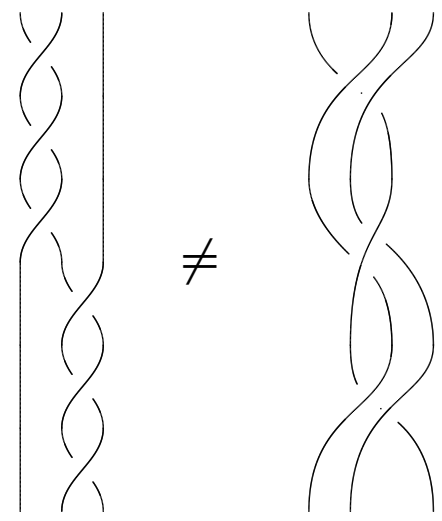

Indeed we have that the required equality of braids for the first hexagonal axiom can never hold for $n \geq 1$. We check the positive powers of $c$ and note that the case for the negative powers is shown similarly. For $c^{\prime}=c^{2 n+1}$ the braid inequality underlying the legs of the hexagonal diagram, in terms of the standard braid generators, is $\sigma_{1}^{2 n+1} \sigma_{2}^{2 n+1} \neq \sigma_{1} \sigma_{2}\left(\sigma_{2} \sigma_{1} \sigma_{1} \sigma_{2}\right)^{n}$. It is easy to see this inequality since the semigroup of positive braids embeds into the braid group of the same number of strands, as shown in Garside [8]. Thus any two positive braids are equivalent in the braid group if and only if they are equivalent in the positive semigroup, that is, related by a chain of braid relations. For three strand braids the only possible braid relation is the standard $\sigma_{1} \sigma_{2} \sigma_{1}=\sigma_{2} \sigma_{1} \sigma_{2}$. Note that in the braid words representing the three strand braids 
in question there are no instances of either side of this relation, and so both are in a unique positive form, and so clearly not equal.

If $\mathcal{V}$ is braided then we can define additional structure on $\mathcal{V}$-Cat. The two classic structures are duality and tensor product.

2.7 Definition First there is a left opposite of a $\mathcal{V}$-category which has $\left|\mathcal{A}^{\mathrm{op}}\right|=|\mathcal{A}|$ and $\mathcal{A}^{\mathrm{op}}\left(A, A^{\prime}\right)=\mathcal{A}\left(A^{\prime}, A\right)$. The composition morphisms are given by

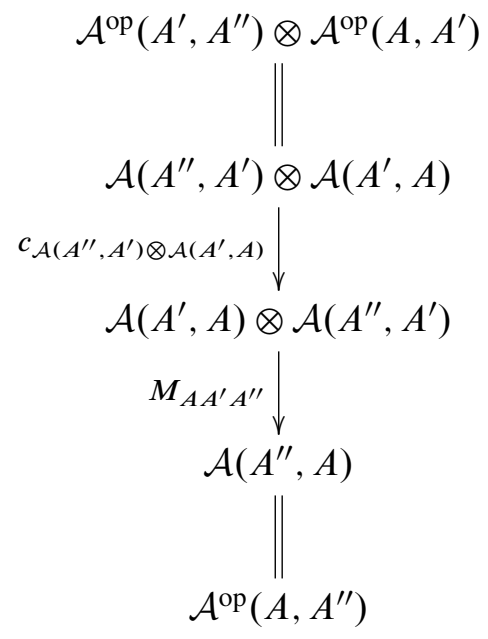

The axiom for associativity of the composition morphisms in $\mathcal{A}^{\text {op }}$ holds due to the naturality of the braiding, the axiom for $M$ in $\mathcal{A}$, and the commutativity of a pentagonal diagram. This latter commutes since the braids underlying its legs are the two sides of the braid relation, also known as the Yang-Baxter equation. The unit morphisms in $\mathcal{A}^{\mathrm{op}}$ are the same as the original $j_{A}: I \rightarrow \mathcal{A}(A, A)=\mathcal{A}^{\mathrm{op}}(A, A)$. The unit axioms are obeyed due to the fact that $c_{I A}=c_{A I}=1_{A}$.

The right opposite denoted $\mathcal{A}^{\text {po }}$ is given by the same definition of composition and unit morphisms, but using $c^{-1}$.

The two opposites take a $\mathcal{V}$-functor $F$ to its own function on objects but with $F_{A A^{\prime}}^{\mathrm{op}}=$ $F_{A A^{\prime}}^{\mathrm{po}}=F_{A^{\prime} A}$. It is easy to check that thus both opposites are functorial. That the image of a $\mathcal{V}$-functor under the opposites is still a $\mathcal{V}$-functor is due to the naturality of $c$.

It is clear from this definition that $\left(\mathcal{A}^{\mathrm{po}}\right)^{\mathrm{op}}=\left(\mathcal{A}^{\mathrm{op}}\right)^{\mathrm{po}}=\mathcal{A}$. It is also clear that $\left(\mathcal{A}^{\mathrm{op}}\right)^{\mathrm{op}} \neq \mathcal{A}$ in general unless $c$ is a symmetry, and the same is true for the right opposite.

Algebraic ${ }^{3} \mathcal{G}$ Geometric Topology, Volume 7 (2007) 
2.8 Definition The second structure is a tensor product for $\mathcal{V}$-Cat, that is, a 2-functor $\otimes: \mathcal{V}-$ Cat $\times \mathcal{V}-$ Cat $\rightarrow \mathcal{V}-$ Cat

(In previous papers we have denoted the product(s) in $\mathcal{V}$-Cat with a superscript (1) in parentheses, but here it will be understood by context. The superscript (1) will still be used to denote that a given natural transformation is in $\mathcal{V}$-Cat.) The product of two $\mathcal{V}$-categories $\mathcal{A}$ and $\mathcal{B}$ has $|\mathcal{A} \otimes \mathcal{B}|=|\mathcal{A}| \times|\mathcal{B}|$ and $(\mathcal{A} \otimes \mathcal{B})\left((A, B),\left(A^{\prime}, B^{\prime}\right)\right)=$ $\mathcal{A}\left(A, A^{\prime}\right) \otimes \mathcal{B}\left(B, B^{\prime}\right)$. The unit morphisms for the product $\mathcal{V}$-categories are the composites

$$
I \cong I \otimes I \underset{j_{A} \otimes j_{B}}{\longrightarrow} \mathcal{A}(A, A) \otimes \mathcal{B}(B, B)
$$

The composition morphisms

$$
\begin{aligned}
M_{(A, B)\left(A^{\prime}, B^{\prime}\right)\left(A^{\prime \prime}, B^{\prime \prime}\right)}:(\mathcal{A} \otimes \mathcal{B})\left(\left(A^{\prime}, B^{\prime}\right),\left(A^{\prime \prime}, B^{\prime \prime}\right)\right) \otimes(\mathcal{A} \otimes \mathcal{B})\left((A, B),\left(A^{\prime}, B^{\prime}\right)\right) & \\
\longrightarrow & (\mathcal{A} \otimes \mathcal{B})\left((A, B),\left(A^{\prime \prime}, B^{\prime \prime}\right)\right)
\end{aligned}
$$

may be given canonically by

$$
\begin{aligned}
& (\mathcal{A} \otimes \mathcal{B})\left(\left(A^{\prime}, B^{\prime}\right),\left(A^{\prime \prime}, B^{\prime \prime}\right)\right) \otimes(\mathcal{A} \otimes \mathcal{B})\left((A, B),\left(A^{\prime}, B^{\prime}\right)\right) \\
& \left(\mathcal{A}\left(A^{\prime}, A^{\prime \prime}\right) \otimes \mathcal{B}\left(B^{\prime}, B^{\prime \prime}\right)\right) \otimes\left(\mathcal{A}\left(A, A^{\prime}\right) \otimes \mathcal{B}\left(B, B^{\prime}\right)\right) \\
& \left(1 \otimes \alpha^{-1}\right) \circ \alpha \\
& \mathcal{A}\left(A^{\prime}, A^{\prime \prime}\right) \otimes\left(\left(\mathcal{B}\left(B^{\prime}, B^{\prime \prime}\right) \otimes \mathcal{A}\left(A, A^{\prime}\right)\right) \otimes \mathcal{B}\left(B, B^{\prime}\right)\right) \\
& 1 \otimes\left(c_{\mathcal{B}\left(B^{\prime}, B^{\prime \prime}\right) \mathcal{A}\left(A, A^{\prime}\right)} \otimes 1\right) \\
& \mathcal{A}\left(A^{\prime}, A^{\prime \prime}\right) \otimes\left(\left(\mathcal{A}\left(A, A^{\prime}\right) \otimes \mathcal{B}\left(B^{\prime}, B^{\prime \prime}\right)\right) \otimes \mathcal{B}\left(B, B^{\prime}\right)\right) \\
& \alpha^{-1} \circ(1 \otimes \alpha) \downarrow \\
& \left(\mathcal{A}\left(A^{\prime}, A^{\prime \prime}\right) \otimes \mathcal{A}\left(A, A^{\prime}\right)\right) \otimes\left(\mathcal{B}\left(B^{\prime}, B^{\prime \prime}\right) \otimes \mathcal{B}\left(B, B^{\prime}\right)\right) \\
& M_{A A^{\prime} A^{\prime \prime}} \otimes M_{B B^{\prime} B^{\prime \prime}} \\
& \mathcal{A}\left(A, A^{\prime \prime}\right) \otimes \mathcal{B}\left(B, B^{\prime \prime}\right) \\
& (\mathcal{A} \otimes \mathcal{B})\left((A, B),\left(A^{\prime \prime}, B^{\prime \prime}\right)\right)
\end{aligned}
$$


That, in general, $(\mathcal{A} \otimes \mathcal{B})^{\mathrm{op}} \neq \mathcal{A}^{\mathrm{op}} \otimes \mathcal{B}^{\mathrm{op}}$ unless $c$ is a symmetry follows from the braid inequality

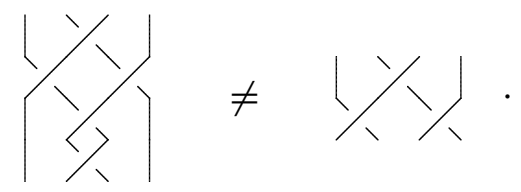

Now consider more carefully the morphisms of $\mathcal{V}$ which make up the composition morphism for a tensor product of enriched categories, especially those which accomplish the "middle four interchange" (see Kelly [10]) of the interior hom-objects, that is, all but the last pair of instances of the original composition $M$. In the symmetric case, any other combination of instances of $\alpha$ and $c$ with the same domain and range would be equal, due to symmetric coherence. In the merely braided case, there at first seems to be a much larger range of available choices. Candidates for composition morphisms would seem to be those defined using any braid $b \in B_{4}$ such that $\sigma(b)=\left(\begin{array}{ll}2 & 3\end{array}\right)$.

Thus a candidate for a new monoidal structure on $\mathcal{V}$-Cat could be given by the same canonical choices for objects, hom-objects, and unit morphisms as in Definition 2.8 but with alternate composition morphisms. The composition morphisms would be defined as above, but with the middle four interchange denoted $\eta_{(b)}$ given by a series of instances of $\alpha$ and $c$ such that the underlying braid is $b$. Thus we might define $M_{(A, B)\left(A^{\prime}, B^{\prime}\right)\left(A^{\prime \prime}, B^{\prime \prime}\right)}=\left(M_{A A^{\prime} A^{\prime \prime}} \otimes M_{B B^{\prime} B^{\prime \prime}}\right) \circ \eta_{(b)}$. That $M_{A A^{\prime} A^{\prime \prime}} \otimes M_{B B^{\prime} B^{\prime \prime}}$ will have the correct domain on which to operate is guaranteed by the permutation condition on $b$.

Two important axioms that must hold for a proposed alternate monoidal structure on $\mathcal{V}$-Cat are associativity of composition $M$ (inside the proposed tensor product of two $\mathcal{V}$-categories) and $\mathcal{V}$-functoriality of the associator $\alpha$ (so that there exists an associator for the proposed tensor product). For the associativity of composition to hold the following diagram must commute, where the first vertex is

$\left((\mathcal{A} \otimes \mathcal{B})\left(\left(A^{\prime \prime}, B^{\prime \prime}\right),\left(A^{\prime \prime \prime}, B^{\prime \prime \prime}\right)\right) \otimes(\mathcal{A} \otimes \mathcal{B})\left(\left(A^{\prime}, B^{\prime}\right),\left(A^{\prime \prime}, B^{\prime \prime}\right)\right)\right) \otimes(\mathcal{A} \otimes \mathcal{B})\left((A, B),\left(A^{\prime}, B^{\prime}\right)\right)$ and the last bullet represents $(\mathcal{A} \otimes \mathcal{B})\left((A, B),\left(A^{\prime \prime \prime}, B^{\prime \prime \prime}\right)\right)$.

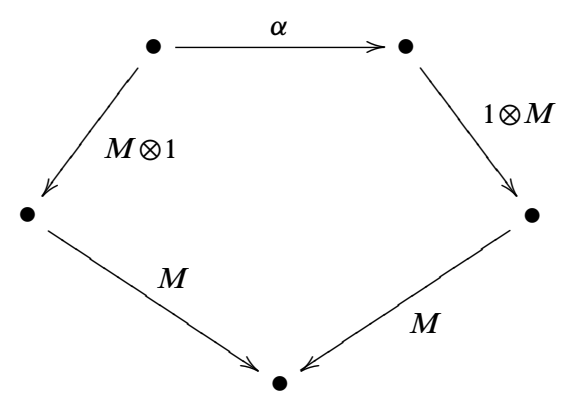

Algebraic 83 Geometric Topology, Volume 7 (2007) 
This means that the exterior of the following expanded diagram is required to commute. We leave out some parentheses for clarity and denote the middle four interchange by $\eta_{(b)}$ (perhaps composed with some associators). Also for convenience we write $X=\mathcal{A}\left(A, A^{\prime}\right), X^{\prime}=\mathcal{A}\left(A^{\prime}, A^{\prime \prime}\right), X^{\prime \prime}=\mathcal{A}\left(A^{\prime \prime}, A^{\prime \prime \prime}\right), Y=\mathcal{B}\left(B, B^{\prime}\right), Y^{\prime}=\mathcal{B}\left(B^{\prime}, B^{\prime \prime}\right)$ and $Y^{\prime \prime}=\mathcal{B}\left(B^{\prime \prime}, B^{\prime \prime \prime}\right)$.

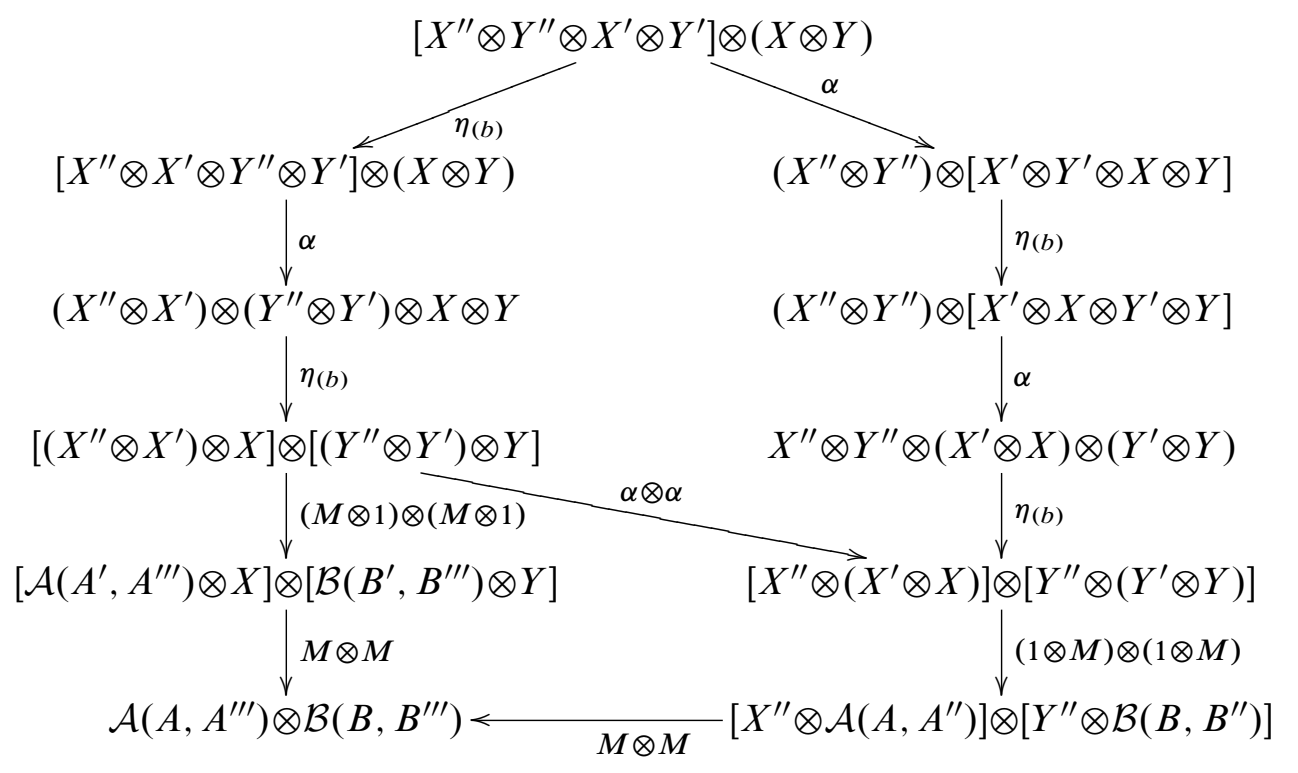

The bottom region commutes by the associativity axioms for $\mathcal{A}$ and $\mathcal{B}$. We are left needing to show that the underlying braids are equal for the two legs of the upper region. In Example 2.9 we give some examples of the underlying braids of the left and right legs for various choices of $b$. By inspection of the diagram these left and right underlying braids are the six-strand braids we denote respectively $L b$ and $R b$. Recall from the introduction that the requirement that $L b=R b$ is called internal associativity. The first example for $b$ is the one used in the original definition of $\mathcal{A} \otimes \mathcal{B}$ given above.

\subsection{Example}

$$
\begin{aligned}
& b_{(1)}=b_{0^{+}}=\mid \text {久 }\left|; \quad L b_{(1)}=\right| \% \biguplus|=| / \% \mid=R b_{(1)} . \\
& b_{(2)}=\mid \frac{y}{y} \\
& L b_{(2)}=\mid 1 / 2
\end{aligned}
$$




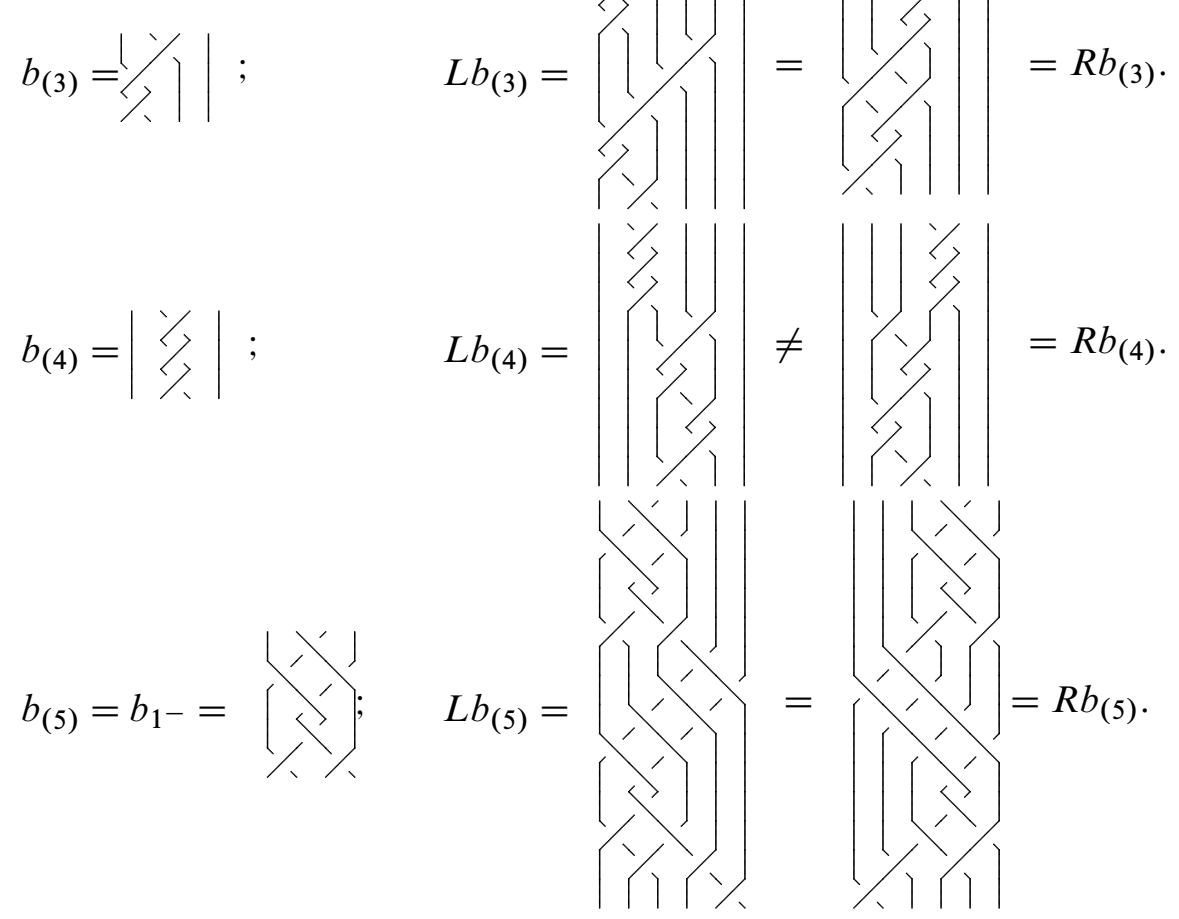

2.10 Remark Before turning to check on $\mathcal{V}$-functoriality of the associator, we note that $b_{(3)}$ is the braid underlying the composition morphism of the product category $\left(\mathcal{A}^{\mathrm{op}}\right)^{\mathrm{op}} \otimes \mathcal{B}$ where the product is defined using $b_{(1)}$. This provides the hint that the two derived braids $L b_{(3)}, R b_{(3)} \in B_{6}$ are equal because of the fact that the opposite of a $\mathcal{V}$-category is a valid $\mathcal{V}$-category. In fact we can describe sufficient conditions for $L b$ to be equivalent to $R b$ by describing the braids $b$ that underlie the composition morphism of a product category given generally by $\left(\left(\left(\mathcal{A}^{\mathrm{op}}\right)^{\cdots \mathrm{op}} \otimes\left(\mathcal{B}^{\mathrm{op}}\right)^{\cdots \mathrm{op}}\right)^{\mathrm{op}}\right)^{\cdots \text { op }}$ where the number of op exponents is arbitrary in each position.

Those braids are alternately described as lying in $H \sigma_{2} K^{\prime} \subset B_{4}$ where $H$ is the cyclic subgroup generated by the braid $\sigma_{2} \sigma_{1} \sigma_{3} \sigma_{2}$ and $K^{\prime}$ is the subgroup generated by the two generators $\left\{\sigma_{1}, \sigma_{3}\right\}$. The latter subgroup $K^{\prime}$ is isomorphic to $Z \times Z$. The first coordinate corresponds to the number of op exponents on $\mathcal{A}$ and the second component to the number of op exponents on $\mathcal{B}$. Negative integers correspond to the right opposites, po. The power of the element of $H$ corresponds to the number of op exponents on the product of the two enriched categories, that is, the number of op exponents outside the parentheses. That $b \in H_{2} \sigma_{2} K^{\prime}$ implies $L b=R b$ follows from 
the fact that the composition morphisms belonging to the opposite of a $\mathcal{V}$-category obey the pentagon axiom. An exercise of some value is to check consistency of the definitions by constructing an inductive proof of the implication based on braid group generators. This is not a necessary condition for $L b=R b$, since for example the equation holds for $b=\left(\sigma_{2} \sigma_{1} \sigma_{3} \sigma_{2}\right)^{n}$, but it may be when the additional requirement that $\sigma(b)=(23)$ is added. More work needs to be done to determine the necessary conditions and to study the structure and properties of the braids that meet these conditions. Of course we will see shortly that when certain unit conditions are obeyed then there is a necessary and sufficient condition.

$\mathcal{V}$-functoriality of the associator is necessary because here we have a 2-natural transformation $\alpha^{(1)}$. This means we have a family of $\mathcal{V}$-functors indexed by triples of $\mathcal{V}$-categories. On objects

$$
\alpha_{\mathcal{A B C}}^{(1)}((A, B), C)=(A,(B, C)) .
$$

In order to guarantee that $\alpha^{(1)}$ obey the coherence pentagon for hom-object morphisms, we define it to be based upon $\alpha$ in $\mathcal{V}$. This means precisely that

$$
\begin{aligned}
\alpha_{\mathcal{A B C}}^{(1)}((A, B), C)\left(\left(A^{\prime}, B^{\prime}\right), C^{\prime}\right) \\
:[(\mathcal{A} \otimes \mathcal{B}) \otimes \mathcal{C}]\left(((A, B), C),\left(\left(A^{\prime}, B^{\prime}\right), C^{\prime}\right)\right) \\
\longrightarrow[\mathcal{A} \otimes(\mathcal{B} \otimes \mathcal{C})]\left((A,(B, C)),\left(A^{\prime},\left(B^{\prime}, C^{\prime}\right)\right)\right)
\end{aligned}
$$

is defined to be

$$
\begin{aligned}
\alpha_{\mathcal{A}\left(A, A^{\prime}\right) \mathcal{B}\left(B, B^{\prime}\right) \mathcal{C}\left(C, C^{\prime}\right)}:\left(\mathcal{A}\left(A, A^{\prime}\right) \otimes \mathcal{B}\left(B, B^{\prime}\right)\right) \otimes \mathcal{C}\left(C, C^{\prime}\right) & \\
& \longrightarrow \mathcal{A}\left(A, A^{\prime}\right) \otimes\left(\mathcal{B}\left(B, B^{\prime}\right) \otimes \mathcal{C}\left(C, C^{\prime}\right)\right) .
\end{aligned}
$$

This definition guarantees that the $\alpha^{(1)}$ pentagons for objects and for hom-objects commute: the first trivially and the second by the fact that the $\alpha$ pentagon commutes in $\mathcal{V}$. We must also check for $\mathcal{V}$-functoriality. The unit axioms are trivial - we consider the more interesting axiom. The following diagram must commute, where the first vertex is

$$
[(\mathcal{A} \otimes \mathcal{B}) \otimes \mathcal{C}]\left(\left(\left(A^{\prime}, B^{\prime}\right), C^{\prime}\right),\left(\left(A^{\prime \prime}, B^{\prime \prime}\right), C^{\prime \prime}\right)\right) \otimes[(\mathcal{A} \otimes \mathcal{B}) \otimes \mathcal{C}]\left(((A, B), C),\left(\left(A^{\prime}, B^{\prime}\right), C^{\prime}\right)\right)
$$

and the last vertex is

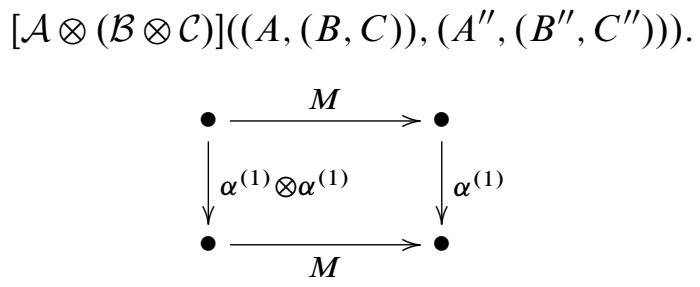

Algebraic 83 Geometric Topology, Volume 7 (2007) 
This means that the exterior of the following expanded diagram is required to commute. We leave out some parentheses for clarity and denote the middle four interchange by $\eta_{(b)}$ (perhaps composed with some associators). Also for convenience we write $X=\mathcal{A}\left(A^{\prime}, A^{\prime \prime}\right), Y=\mathcal{B}\left(B^{\prime}, B^{\prime \prime}\right), Z=\mathcal{C}\left(C^{\prime}, C^{\prime \prime}\right), X^{\prime}=\mathcal{A}\left(A, A^{\prime}\right), Y^{\prime}=\mathcal{B}\left(B, B^{\prime}\right)$ and $Z^{\prime}=\mathcal{C}\left(C, C^{\prime}\right)$

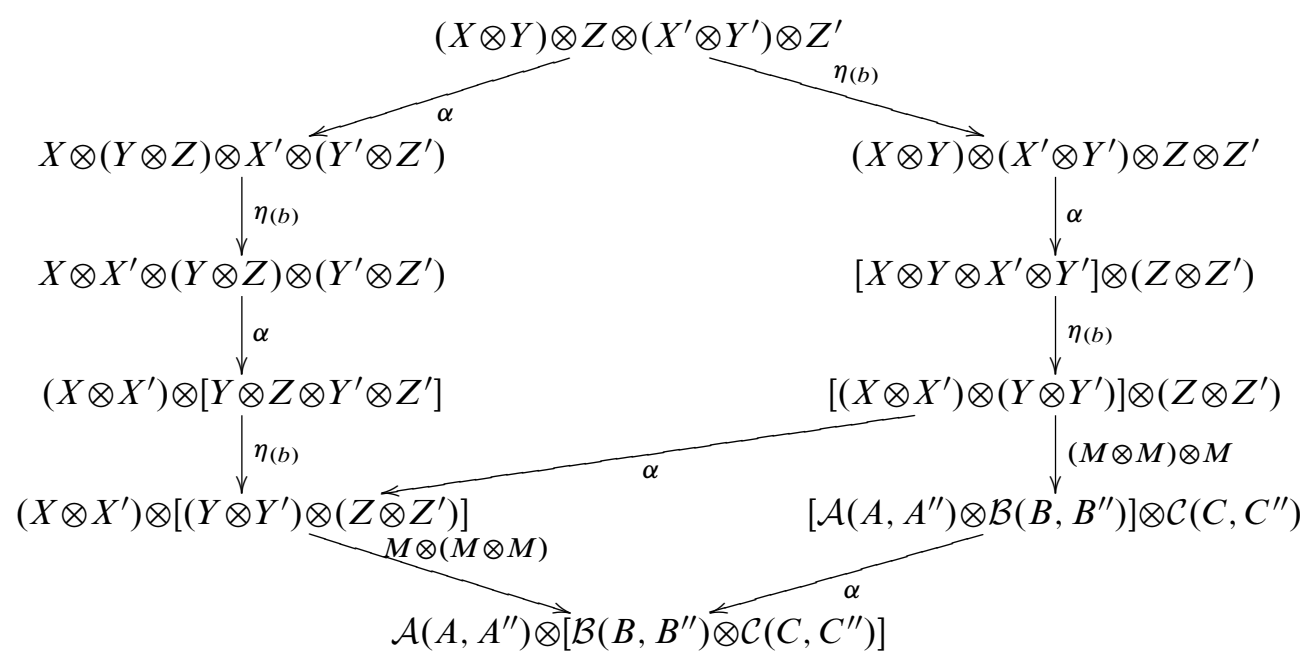

The bottom quadrilateral commutes by naturality of $\alpha$. We are left needing to show that the underlying braids are equal for the two legs of the upper region. In Example 2.11 we give some examples of the underlying braids of the left and right legs for the same choices of $b$ as shown in Example 2.9. By inspection of the diagram these left and right underlying braids are the six-strand braids we denote respectively $L^{\prime} b$ and $R^{\prime} b$. Recall from the introduction that the requirement that $L^{\prime} b=R^{\prime} b$ is called external associativity. The first braid is the one used in the original definition of $\mathcal{A} \otimes \mathcal{B}$ given above.

\subsection{Example}

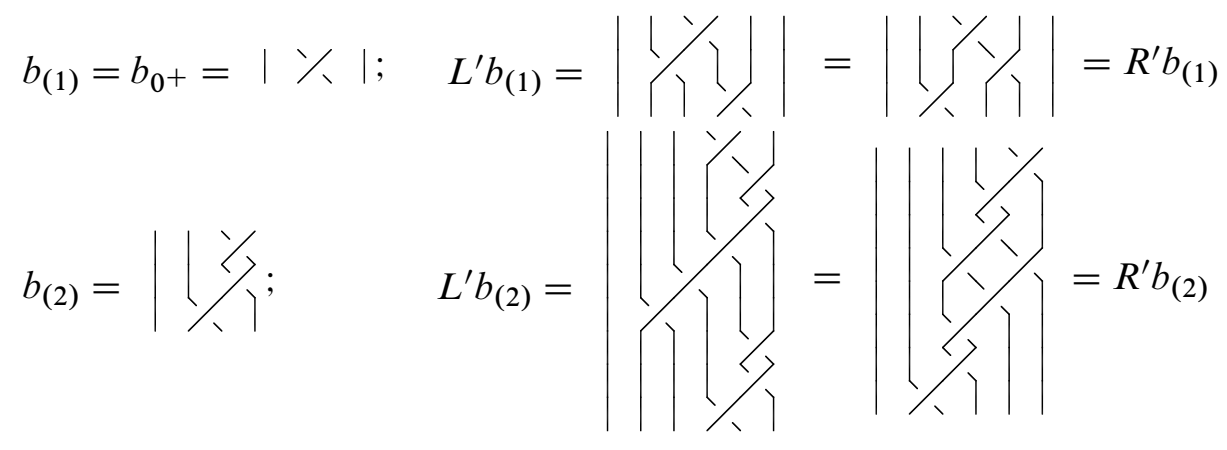

Algebraic $8 \mathcal{G}$ Geometric Topology, Volume 7 (2007) 
$b_{(3)}=\langle/ \%|$

$b_{(4)}=|y|$
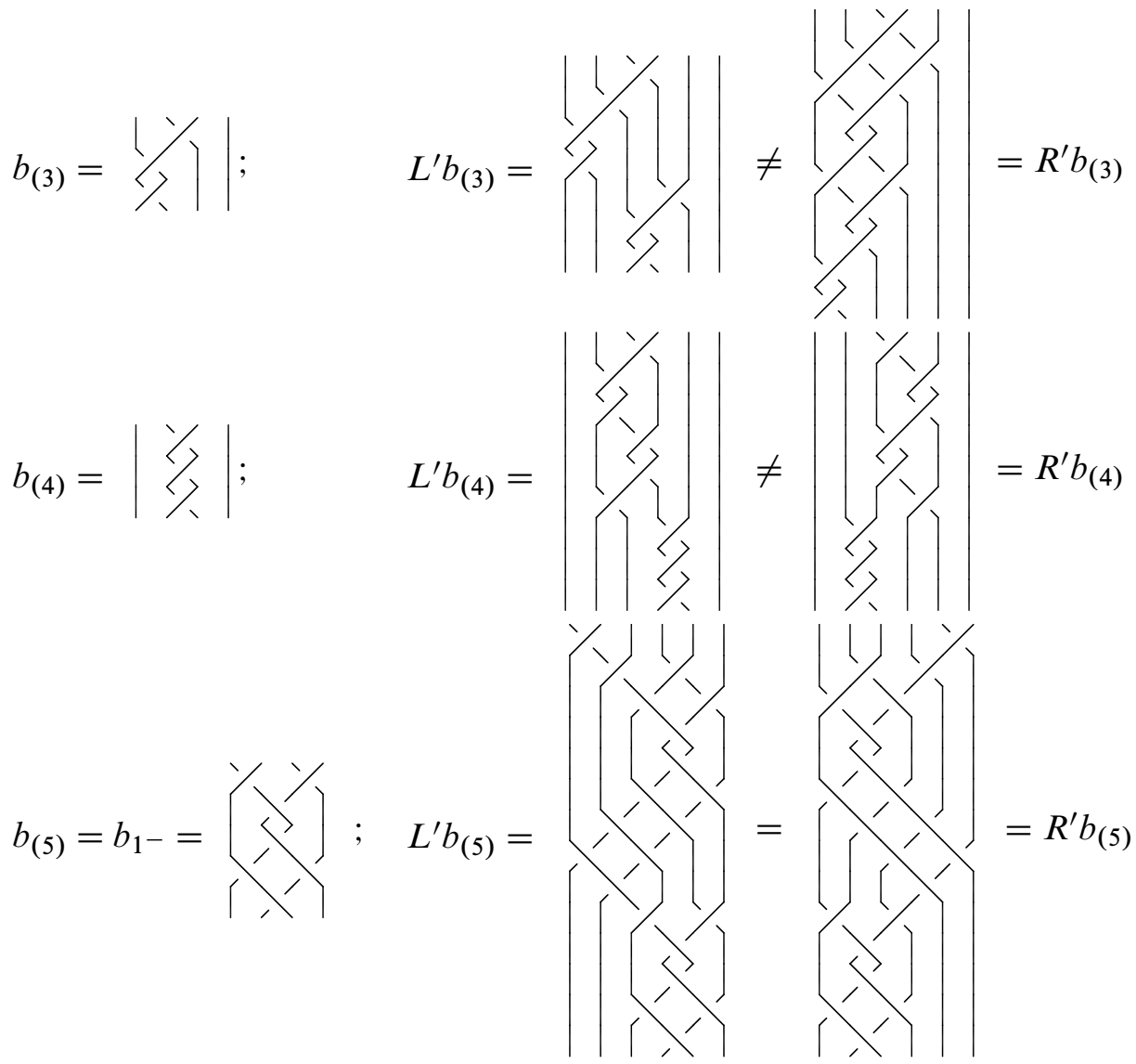

2.12 Remark A comparison with Example 2.9 is of interest. Braids $b_{(2)}$ and $b_{(3)}$ are 180 degree rotations of each other. Notice that the second braid in Example 2.11 leads to an equality that is actually the same as for the third braid in Example 2.9. To see this the page can be rotated by 180 degrees. Similarly, the inequality preventing braid $b_{(2)}$ from yielding an associative composition morphism is the 180 degree rotation of the inequality preventing braid $b_{(3)}$ from yielding a $\mathcal{V}$-functorial associator. Braid $b_{(1)}$ and braid $b_{(5)}$ are each their own 180 degree rotation (we took advantage of the latter fact in drawing $L^{\prime} b_{(5)}$ and $R^{\prime} b_{(5)}$ above), and the two braids proving each to be the underlying braid of an associative composition morphism are 180 degree rotations of the two which show each to yield a $\mathcal{V}$-functorial associator. Braid $b_{(4)}$ is its own 180 degree rotation, and the two braids preventing it from yielding an associative composition morphism are 180 degree rotations of the two that obstruct it from being yielding a $\mathcal{V}$-functorial associator. Thus there is a certain kind of duality between 
the requirements of associativity of the enriched composition and the functoriality of the associator. The full meaning of this duality becomes more clear in the study of (enrichment over) iterated monoidal categories as in Forcey [6], where we see that in a braided category two potentially different tensor products have collapsed into one.

If we were considering a strictly associative monoidal category $\mathcal{V}$ then the condition of a $\mathcal{V}$-functorial associator would become a condition of a well defined composition morphism.

The unit axioms required of the tensor product of two enriched categories are satisfied in general only if dropping either the first two or the last two strands of $b$ leaves again the identity on two strands. (This is also due to the naturality of compositions of $\alpha$ and $c$ and the unit axioms obeyed by $\mathcal{A}$ and $\mathcal{B}$.) Recall that we refer to this as the internal unit condition.

The canonical choice for the unit in $\mathcal{V}$-Cat is the enriched category $\mathcal{I}$ which has only one object denoted 0 and for which $\mathcal{I}(0,0)=I$, the unit in $\mathcal{V}$. For the unit $\mathcal{V}$-category $\mathcal{I}$ to be indeed a unit for the tensor product in question requires that in the underlying braid of the middle four interchange dropping either the first and third strand or the second and fourth strand leaves the identity on two strands. Recall that we refer to this as the external unit condition. For a careful demonstration of this see [6], keeping in mind that the interchange $\eta$ described there corresponds to the middle four interchange here. Note that the unit conditions are not met by $b_{(2)}$ and $b_{(3)}$ in the above examples.

2.13 Definition An interchanging unital braid on four strands is one for which the permutation associated to the braid is (23), for which both $L b=R b$ and $L^{\prime} b=R^{\prime} b$ in $B_{6}$, and for which the unit conditions are satisfied: deleting any one of the pairs of strands $(1,2) ;(3,4) ;(1,3)$ or $(2,4)$ results in the 2 strand identity braid. An interchange candidate braid is an element of $B_{4}$ which has the correct permutation and obeys the unit conditions.

Note that this definition describes precisely what needs to be true of a braid $b$ in order that it can arise as the underlying braid of the middle four interchange of the composition morphism of the tensor product of enriched categories over an arbitrary braided category $\mathcal{V}$. Here we are restricting our attention to monoidal structures on $\mathcal{V}$ Cat with the canonical choices described in Definition 2.8 for the objects, hom-objects and unit morphisms of a tensor product of enriched categories. Also let the associator for that monoidal structure be based upon the associator in the braided category $\mathcal{V}$, and the unit for that monoidal structure be the canonical choice of the enriched category $\mathcal{I}$. 
2.14 Lemma Given an arbitrary braided category $\mathcal{V}$, let a monoidal structure on $\mathcal{V}-$ Cat be assumed to have the canonical choices described in Definition 2.8 for the objects, hom-objects and unit morphisms of a tensor product of enriched categories. Also let the associator for that monoidal structure be based upon the associator in the braided category $\mathcal{V}$, and the unit for that monoidal structure be the canonical choice of the enriched category $\mathcal{I}$. Then a four-strand braid $b$ satisfies $L^{\prime} b=R^{\prime} b$ if it can arise as the underlying braid of the middle four interchange of the composition morphism of the tensor product of enriched categories in any such monoidal structure.

Proof Simply having a valid tensor product in $\mathcal{V}$-Cat for a specific braided category $\mathcal{V}$, with a middle four interchange built out of instances of the the associators and the braiding, does not imply that the underlying braid of the middle four interchange is interchanging (e.g. the braiding might be a symmetry or the composition might be a coequalizer). However if $b$ underlies a middle four interchange which gives a tensor product of $\mathcal{V}$-categories which is valid for an arbitrary braided base, then we can get our result by choosing the example of the free braided category on one object with duals, denoted $C_{1,2}$ as in Baez [1].

Notice that we need more structure than just the free braided category on one object. This is because we are only given the equality in $\mathcal{V}$ implied by the diagram for $\mathcal{V}-$ functoriality of $\alpha^{(1)}$; this equality is that of two compositions of braidings each with a tensor product of instances of $M$ attached. Specifically $M \otimes M \otimes M$ follows each of the compositions of braidings. Let the generating objects of $C_{1,2}$ be $x$ and its dual $x^{*}$. Recall that the objects are then strings of these generators and the morphisms are tangles with the number of inputs the length of the domain and the number of outputs the length of the range. The braiding is the same as described in Joyal and Street [9] for the free braided category; the tangle formed by crossing all the strands corresponding to an object $A$ (one strand for each generator in the string) simultaneously with all those of $B$. We can find enriched categories over $C_{1,2}$ since there are monoids in $C_{1,2}$, recalling that monoids are one-object enriched categories. To see the braid equality $L^{\prime} b=R^{\prime} b$ we can choose the monoid $\mathcal{X}=x \otimes x^{*}$. Then the composition morphism is given by

$$
M=1_{x} \otimes e \otimes 1_{x^{*}}: x \otimes x^{*} \otimes x \otimes x^{*} \rightarrow x \otimes x^{*}
$$

(where $e$ is the counit) which corresponds to the tangle

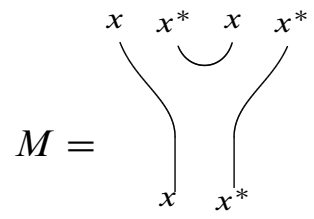

Algebraic 83 Geometric Topology, Volume 7 (2007) 
Now the equality implied by $\mathcal{V}$-functoriality of the associator $\alpha_{\mathcal{X} \mathcal{X}}^{(1)}$ is a tangle equality which is formed by starting with doubled versions of the braids $L^{\prime} b$ and $R^{\prime} b$ (doubled since there is a strand for $x$ and $x^{*}$ ). Then both tangles are finished with $M \otimes M \otimes M$, that is, three copies of the above tangle for $M$ attached. For example, here is the left hand side (left leg) of the tangle equality for the braid $b_{(1)}$ that is implied by the $\mathcal{V}$-functoriality of the associator. Compare to $L^{\prime} b_{(1)}$ above.

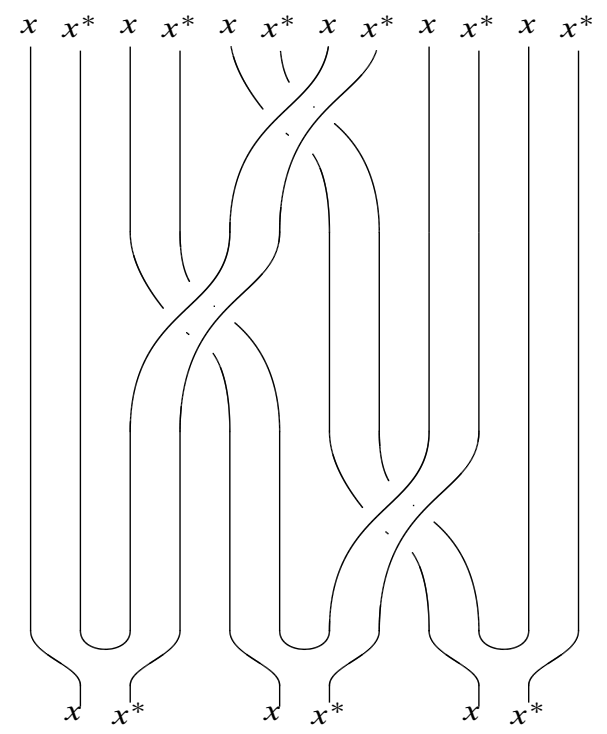

The right hand side is similarly drawn, with a doubled version of $R^{\prime} b_{(1)}$ followed by three copies of $M$.

That the two tangles are equal implies that their corresponding sub-tangles are equal; specifically that their sub-tangles formed by deleting all but the input strands $1,3,5,8,10$, and 12 (which comprise all six output strands) are equal. These sub-tangles are $L^{\prime} b$ and $R^{\prime} b$ respectively.

The question now is whether there are braids underlying the composition of a product of enriched categories besides the braids $b_{(1)}$ and $b_{(5)}$ above (and their inverses) which fulfill all obligations. The answer is yes. To find interchanging braids we iteratively build new monoidal structures from the standard ones, using the duality structure that exists on $\mathcal{V}$-Cat. By $\mathcal{A}^{\mathrm{op}^{n}}$ we denote the $n^{\text {th }}$ (left) opposite of $\mathcal{A}$. By $\otimes$ and $\otimes^{\prime}$ we denote the standard tensor products defined respectively with braid $b_{(1)}$ and its inverse underlying the middle four interchange.

2.15 Theorem The tensor product of enriched categories given by

$$
\mathcal{A} \otimes_{1}-\mathcal{B}=\left(\mathcal{A}^{\mathrm{op}} \otimes^{\prime} \mathcal{B}^{\mathrm{op}}\right)^{\mathrm{po}}
$$

Algebraic $8 \mathcal{G}$ Geometric Topology, Volume 7 (2007) 
is a valid monoidal product on $\mathcal{V}-$ Cat. Furthermore, so are the tensor products

$$
\mathcal{A} \otimes_{n^{-}} \mathcal{B}=\left(\mathcal{A}^{\mathrm{op}^{n}} \otimes^{\prime} \mathcal{B}^{\mathrm{op}^{n}}\right)^{\mathrm{po}^{n}}
$$

as well as those with underlying braids that are the inverses of these, denoted

$$
\mathcal{A} \otimes_{n}+\mathcal{B}=\left(\mathcal{A}^{\mathrm{po}^{n}} \otimes \mathcal{B}^{\mathrm{po}^{n}}\right)^{\mathrm{op}^{n}} .
$$

Proof The first tensor product is mentioned alone since the middle four interchange in its composition morphism has the underlying braid shown above as braid $b_{(5)}$. Thus we have already demonstrated its fitness as a monoidal product. However this can be more efficiently shown just by noting that the category given by the product is certainly a valid enriched category, and that for three operands we have an associator from the isomorphism given by the following:

$$
\begin{aligned}
\alpha_{\mathcal{A B C}}^{1^{-}} & :\left(\mathcal{A} \otimes_{1}-\mathcal{B}\right) \otimes_{1}-\mathcal{C} \\
& =\left(\left(\left(\mathcal{A}^{\mathrm{op}} \otimes^{\prime} \mathcal{B}^{\mathrm{op}}\right)^{\mathrm{po}}\right)^{\mathrm{op}} \otimes^{\prime} \mathcal{C}^{\mathrm{op}}\right)^{\mathrm{po}} \\
& =\left(\left(\mathcal{A}^{\mathrm{op}} \otimes^{\prime} \mathcal{B}^{\mathrm{op}}\right) \otimes^{\prime} \mathcal{C}^{\mathrm{op}}\right)^{\mathrm{po}} \\
& \cong\left(\mathcal{A}^{\mathrm{op}} \otimes^{\prime}\left(\mathcal{B}^{\mathrm{op}} \otimes^{\prime} \mathcal{C}^{\mathrm{op}}\right)\right)^{\mathrm{po}} \\
& =\left(\mathcal{A}^{\mathrm{op}} \otimes^{\prime}\left(\left(\mathcal{B}^{\mathrm{op}} \otimes^{\prime} \mathcal{C}^{\mathrm{op}}\right)^{\mathrm{po}}\right)^{\mathrm{op}}\right)^{\mathrm{po}} \\
& =\mathcal{A} \otimes_{1^{-}}\left(\mathcal{B} \otimes_{1}-\mathcal{C}\right)
\end{aligned}
$$

The associator implicit in this isomorphism is constructed by taking the right opposite of instances of the standard associator for $\otimes^{\prime}$;

$$
\alpha_{\mathcal{A B C}}^{1^{-}}=\left(\alpha_{\mathcal{A}^{\mathrm{op}} \mathcal{B}^{\mathrm{op}} \mathcal{C}^{\mathrm{op}}}^{\prime(1)}\right)^{\mathrm{po}} .
$$

The standard associator for $\otimes^{\prime}$ is identical to the one for $\otimes$. Thus $\alpha^{1^{-}}$is based upon $\alpha$ in $\mathcal{V}$, since the object sets of the domain and range are the usual cartesian products and since

$$
\alpha_{\mathcal{A B C}}^{1_{((A, B), C)\left(\left(A^{\prime}, B^{\prime}\right), C^{\prime}\right)}^{-}}=\alpha_{\mathcal{A}\left(A, A^{\prime}\right) \mathcal{B}\left(B, B^{\prime}\right) \mathcal{C}\left(C, C^{\prime}\right)} .
$$

This new associator is guaranteed to have $\mathcal{V}$-functorial instances since they are the images (under the right opposite) of $\mathcal{V}$-functors.

Inductively this process can be repeated with all the left opposites and right opposites raised to the $n^{\text {th }}$ degree. Recall that the unit $\mathcal{V}$-category $\mathcal{I}$ has only one object 0 and $\mathcal{I}(0,0)=I$, the unit in $\mathcal{V}$. That $\mathcal{I}$ is indeed a unit for the tensor products in question follows from the facts that $\mathcal{I}^{\mathrm{op}}=\mathcal{I}=\mathcal{I}^{\text {po }}$ which are in turn evident from facts $c_{I A}=c_{A I}=1_{A}$. Thus we have that, using any of the above tensor products including the standard ones $\otimes=\otimes_{0^{+}}$and $\otimes^{\prime}=\otimes_{0^{-}}$defined respectively with braid $b_{(1)}$ and its inverse, $\mathcal{V}$-Cat is a monoidal 2 -category. 
The braids underlying these new tensor products are not hard to describe directly. Suppressing the associators, the instances of the braiding forming the middle four interchange for $\otimes_{1}+$ are as follows, using $X^{\prime}, Y^{\prime}, X, Y$ to stand for hom objects as above:

$$
\left(c_{X X^{\prime}} \otimes c_{Y Y^{\prime}}\right) \circ\left(1_{X} \otimes c_{Y X^{\prime}}^{-1} \otimes 1_{Y^{\prime}}\right) \circ\left(c_{\left(X^{\prime} \otimes Y^{\prime}\right)(X \otimes Y)}^{-1}\right),
$$

with underlying braid $b_{(5)}$. Note that $b_{(5)}$ has also been denoted $b_{1^{-}}$. Another is the following braid which underlies $\otimes_{2^{+}}$:

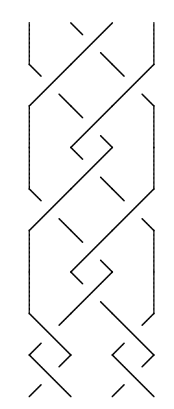

Note that this is precisely the braid $b_{2}+$ shown in the introduction. In fact the construction of the new products leads to the observation that the braid underlying the middle four interchange in the composition for the product $\mathcal{A} \otimes_{n^{ \pm}} \mathcal{B}$ is the previously defined braid $b_{n^{ \pm}}$.

2.16 Remark Note that if in the definition of $\otimes_{n^{ \pm}}$we replace $\otimes^{\prime}$ with $\otimes$ or vice versa, then we have another valid tensor product, but with a braid underlying the middle four interchange in its composition morphisms equivalent to that found in $\otimes_{(n-1)^{ \pm}}$. For example:

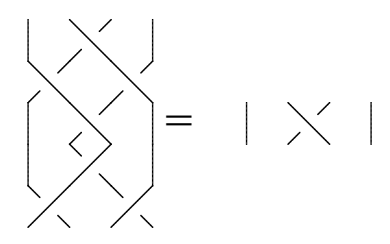

Next we will show that this condition of being equivalent to some $b_{n^{ \pm}}$is necessary for a braid to be interchanging, offer some quick checks to determine when this condition holds, and investigate when the resulting monoidal categories are equivalent. All these steps are best taken in the context of iterated monoidal categories.

\section{2-fold monoidal categories}

In this section we closely follow Balteanu, Fiedorowicz, Schwänzl and Vogt [3] in defining a notion of iterated monoidal category. For those readers familiar with that 
source, note that we vary from their definition only by including associativity up to natural coherent isomorphisms. Thus we begin by reviewing the definition of lax monoidal functor. In our examples using a braided category, however, the natural transformations will all be isomorphisms.

3.1 Definition A lax monoidal functor $(F, \eta): \mathcal{C} \rightarrow \mathcal{D}$ between monoidal categories consists of a functor $F$ such that $F(I)=I$ together with a natural transformation

$$
\eta_{A B}: F(A) \otimes F(B) \rightarrow F(A \otimes B),
$$

which satisfies the following conditions

Internal Associativity: The following diagram commutes

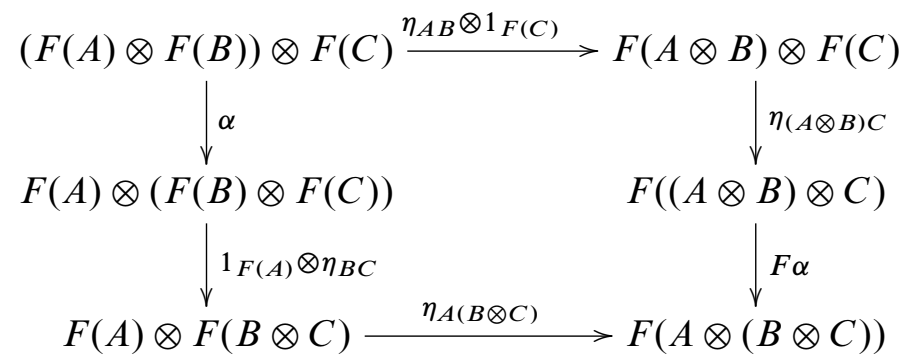

Internal Unit Conditions: $\eta_{A I}=\eta_{I A}=1_{F(A)}$.

Given two monoidal functors $(F, \eta): \mathcal{C} \rightarrow \mathcal{D}$ and $(G, \zeta): \mathcal{D} \rightarrow \mathcal{E}$, we define their composite to be the monoidal functor $(G F, \xi): \mathcal{C} \rightarrow \mathcal{E}$, where $\xi$ denotes the composite

$$
G F(A) \otimes G F(B) \stackrel{\zeta_{F(A) F(B)}}{\longrightarrow} G(F(A) \otimes F(B)) \stackrel{G\left(\eta_{A B}\right)}{\longrightarrow} G F(A \otimes B) .
$$

It is easy to verify that $\xi$ satisfies the internal associativity condition above by subdividing the necessary commuting diagram into two regions that commute by the axioms for $\eta$ and $\zeta$ respectively and two that commute due to their naturality. MonCat is the monoidal category of monoidal categories and monoidal functors, with the usual Cartesian product as in Cat.

A monoidal natural transformation $\theta:(F, \eta) \rightarrow(G, \zeta): \mathcal{D} \rightarrow \mathcal{E}$ is a natural transformation $\theta: F \rightarrow G$ between the underlying ordinary functors of $F$ and $G$ such that the following diagram commutes

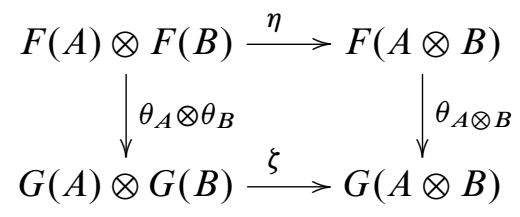

Algebraic 83 Geometric Topology, Volume 7 (2007) 
3.2 Definition A 2-fold monoidal category (with strong associators) is a monoidal category $\left(\mathcal{V}, \otimes_{1}, \alpha^{1}, I\right)$ and a monoidal functor $\left(\otimes_{2}, \eta\right): \mathcal{V} \times \mathcal{V} \rightarrow \mathcal{V}$ which satisfies

External Associativity: the following diagram describes a monoidal natural isomorphism $\alpha^{2}$ in MonCat.

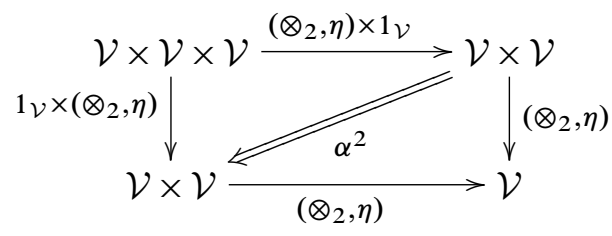

External Unit Conditions: the following diagram commutes in MonCat

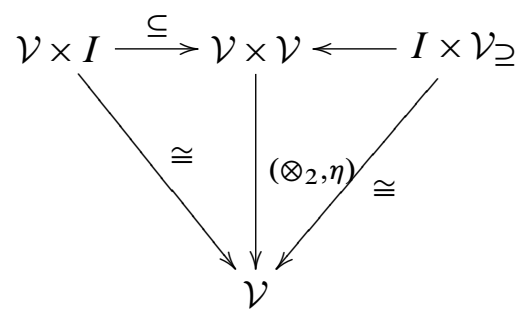

Coherence: The underlying natural transformation $\alpha^{2}$ satisfies the usual coherence pentagon.

Explicitly this means that we are given a second associative binary operation $\otimes_{2}: \mathcal{V} \times$ $\mathcal{V} \rightarrow \mathcal{V}$, for which $I$ is also a two-sided unit. We are also given a natural transformation called the interchange which is the functoriality constraint for $\otimes_{2}$ :

$$
\eta_{A B C D}:\left(A \otimes_{2} B\right) \otimes_{1}\left(C \otimes_{2} D\right) \rightarrow\left(A \otimes_{1} C\right) \otimes_{2}\left(B \otimes_{1} D\right) .
$$

The internal unit conditions for $\otimes_{2}$ as a monoidal functor give $\eta_{A B I I}=\eta_{I I A B}=$ $1_{A \otimes_{2} B}$, while the external unit conditions give $\eta_{A I B I}=\eta_{I A I B}=1_{A \otimes_{1} B}$. The internal associativity condition for $\otimes_{2}$ as a monoidal functor gives the commutative diagram

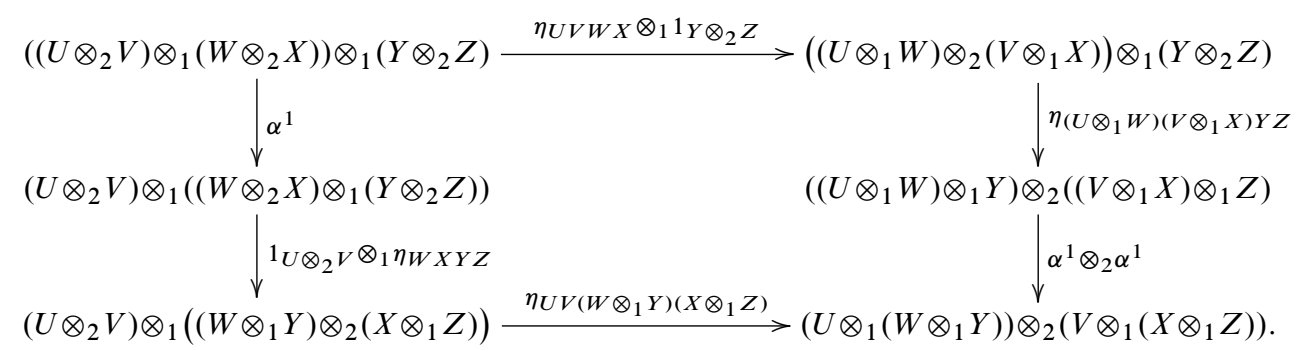

Algebraic $8 \mathcal{G}$ Geometric Topology, Volume 7 (2007) 
The external associativity condition ( $\alpha^{2}$ must be a monoidal natural transformation) gives the commutative diagram:

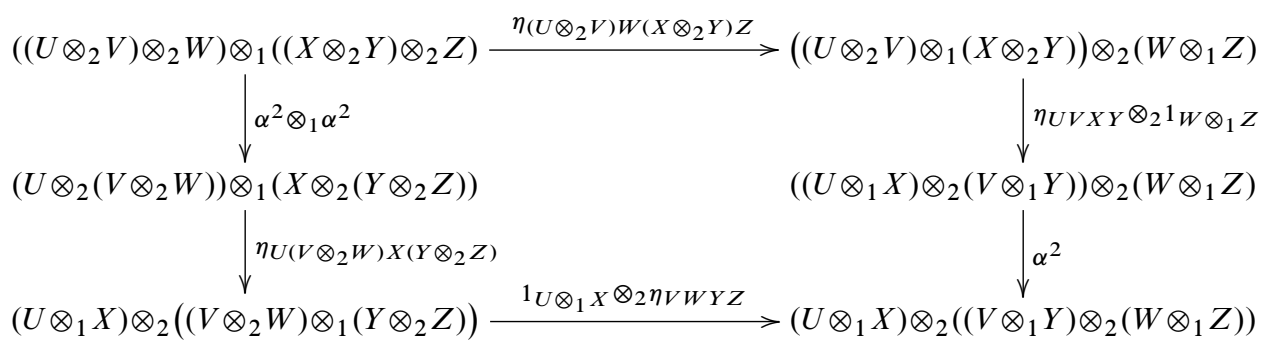

Just as in Balteanu, Fiedorowicz, Schwänzl and Vogt [3] we now define a 2-fold monoidal functor $\left(F, \lambda^{1}, \lambda^{2}\right)$ between 2-fold monoidal categories. It is a functor $F: \mathcal{V} \rightarrow \mathcal{W}$ together with two natural transformations

$$
\lambda_{A B}^{1}: F(A) \otimes_{1} F(B) \rightarrow F\left(A \otimes_{1} B\right) \quad \text { and } \quad \lambda_{A B}^{2}: F(A) \otimes_{2} F(B) \rightarrow F\left(A \otimes_{2} B\right)
$$

satisfying the same associativity and unit conditions as in the case of monoidal functors. In addition we require that the following hexagonal interchange diagram commutes:

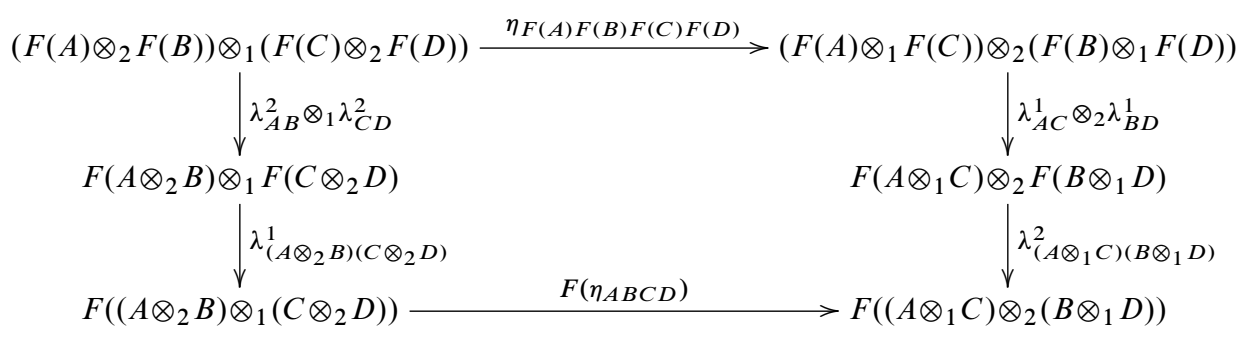

We can now refer to the category 2-MonCat of 2-fold monoidal categories and 2-fold monoidal functors.

The authors of [3] remark that we have natural transformations

$$
\eta_{A I I B}: A \otimes_{1} B \rightarrow A \otimes_{2} B \text { and } \eta_{I A B I}: A \otimes_{1} B \rightarrow B \otimes_{2} A \text {. }
$$

If they had insisted a 2-fold monoidal category be a tensor object in the category of monoidal categories and strictly monoidal functors, this would be equivalent to requiring that $\eta=1$. In view of the above, they note that this would imply $A \otimes_{1} B=$ $A \otimes_{2} B=B \otimes_{1} A$ and similarly for morphisms. This is shown by what is usually referred to as the Eckmann-Hilton argument.

Joyal and Street [9] considered a similar concept to Balteanu, Fiedorowicz, Schwänzl and Vogt's idea of 2-fold monoidal category. The former pair required the natural transformation $\eta_{A B C D}$ to be an isomorphism and showed that the resulting category is a braided monoidal category. As explained in [3], given such a category one obtains an 
equivalent braided monoidal category by ignoring one of the two operations, say $\otimes_{2}$, and defining the braiding for the remaining operation $\otimes_{1}$ to be the composite

$$
A \otimes_{1} B \stackrel{\eta_{I A B I}}{\longrightarrow} B \otimes_{2} A \stackrel{\eta_{B I I A}^{-1}}{\longrightarrow} B \otimes_{1} A .
$$

In [3] it is shown that a 2 -fold monoidal category with $\otimes_{1}=\otimes_{2}=\otimes, \eta$ an isomorphism and

$$
\eta_{A I B C}=\eta_{A B I C}=1_{A \otimes B \otimes C}
$$

is a braided monoidal category with the braiding $c_{B C}=\eta_{I B C I}$.

Also note that for $\mathcal{V}$ braided the interchange given by $\eta_{A B C D}=1_{A} \otimes c_{B C} \otimes 1_{D}$ gives a 2-fold monoidal category where $\otimes_{1}=\otimes_{2}=\otimes$. This interchange has the underlying braid $\sigma_{2} \in B_{4}$. In this setting we ask whether, given a braiding, there are alternate 2-fold monoidal structures on $\mathcal{V}$, with $\otimes_{1}=\otimes_{2}=\otimes$. This is the same question as asking whether there are other interchanging unital braids besides $b_{0^{+}}=b_{(1)}=\sigma_{2}$ and its inverse. To be precise, given a braided category $(\mathcal{V}, \otimes, \alpha, c, I)$ (with strict units, a strong associator $\alpha$, and braiding $c$ ), we ask the central question: For which four-strand braids $b$ does the category $\mathcal{V}$ have in general a coherent 2 -fold monoidal structure, when that structure has $\otimes_{1}=\otimes_{2}=\otimes$ as functors, has $\alpha^{1}=\alpha^{2}=\alpha$ as natural transformations, has strict unit $I$ for both identical tensor products, and has $b$ as the underlying braid of $\eta$ ?

3.3 Lemma Given an arbitrary braided category $\mathcal{V}$, let the 2-fold structure of $\mathcal{V}$ be given by $\otimes_{1}=\otimes_{2}=\otimes$. Then a four-strand braid $b$ is interchanging and unital if and only if any interchange $\eta$ with underlying braid $b$ obeys the axioms of a 2-fold monoidal category.

Proof $L b=R b$ implies the internal associativity axiom of a 2-fold monoidal category, and $L^{\prime} b=R^{\prime} b$ implies the external associativity axiom, as we have foreshadowed with the naming of these braid equalities. This is seen by the coherence theorem for braided categories. The unit axioms for the interchange are also implied by the unit conditions on the braid, described by the fact that deleting certain pairs of strands yields the identity braid. The converse implication is found by letting $\mathcal{V}$ be the free braided category. Then the axioms of a 2-fold monoidal category become precisely the desired braid equalities.

Now we are almost ready to state and prove the main result. First there are a couple of geometric observations to be made about the braids $b_{n^{ \pm}}=\left(\sigma_{2} \sigma_{1} \sigma_{3} \sigma_{2}\right)^{ \pm n} \sigma_{2}^{ \pm 1}\left(\sigma_{1} \sigma_{3}\right)^{\mp n}$. Recall that we refer to the strands of a braid by their initial positions. A sub-braid will refer to the braid resulting from the deletion of a subset of the strands of a braid. 
3.4 Lemma If $n$ is odd then deleting the outer two strands in the braid $b_{n^{ \pm}}$leaves the two strand sub-braid $\sigma_{1}^{ \pm n}$, while deleting the inner two strands gives the sub-braid $\sigma_{1}^{ \pm(n+1)}$. If $n$ is even then deleting the outer two strands in the braid $b_{n^{ \pm}}$leaves the two strand sub-braid $\sigma_{1}^{ \pm(n+1)}$, while deleting the inner two strands gives the sub-braid $\sigma_{1}^{ \pm n}$.

Proof Consider the upper portion of the braid $b_{n^{ \pm}}$given by $\left(\sigma_{2} \sigma_{1} \sigma_{3} \sigma_{2}\right)^{ \pm n}$. The outer two strands and the inner pair of strands both are crossed $\pm n$ times. If $n$ is even then the upper portion is pure and so the next generator $\sigma_{2}^{ \pm 1}$ is applied to the inner two strands. If $n$ is odd then the upper portion has the associated permutation which sends $\left\{\begin{array}{llll}1 & 2 & 3 & 4\end{array}\right\} \rightarrow\left\{\begin{array}{llll}3 & 4 & 1 & 2\end{array}\right\}$ and so the next generator $\sigma_{2}^{ \pm 1}$ is applied to the outer two strands. Note that the lower portion of the braid given by $\left(\sigma_{1} \sigma_{3}\right)^{\mp n}$ contributes no further crossings to either the outer or inner sub-braids.

3.5 Corollary The braids $b_{n^{ \pm}}$and $b_{m^{ \pm}}$are equivalent if and only if $m=n$ and the superscript signs are the same.

Proof For two braids to be equivalent it is necessary that all their corresponding sub-braids be equivalent. If $n, m$ are both odd (or both even) and the signs are the same then the implication is clear by Lemma 3.4. Let $n, m$ be both odd (or both even) with the signs not the same. Then if we assume that $b_{n^{ \pm}}$and $b_{m^{ \pm}}$are equivalent then use of Lemma 3.4 leads to the absurd implication $1=-1$. Let $n$ be odd and $m$ be even with the superscript signs the same. Then if the sub-braids formed by the outer strands are equal we have that $m=n+1$. Then $m+1=n+2 \neq n$ so the inner sub-braids are not equal. Finally let $n$ be odd and $m$ be even with the superscript signs not the same. If the braids are equivalent then $m=-(n+1)$ but both $m$ and $n$ are required to be non-negative, so this is a contradiction.

Now the main result:

3.6 Theorem $A$ braid $b \in B_{4}$ is interchanging and unital if and only if it is equivalent to one of the braids $b_{n^{ \pm}}$.

Proof We will show: $\left[b=b_{n^{ \pm}}\right] \Longrightarrow[b$ gives rise to a middle four interchange for a monoidal structure on $\mathcal{V}$-Cat for arbitrary $\mathcal{V}] \Longrightarrow[b$ interchanging and unital $] \Longrightarrow$ $\left[b=b_{n^{ \pm}}\right]$.

By Theorem 2.15 the middle four interchanges given by suppressing the associators

$$
\eta_{A B C D}=\left(c_{C A}^{\mp n} \otimes c_{D B}^{\mp n}\right) \circ\left(1_{C} \otimes c_{D A}^{ \pm 1} \otimes 1_{B}\right) \circ\left(c_{(A \otimes B)(C \otimes D)}^{ \pm n}\right)
$$


with underlying braid $b_{n^{ \pm}}$, are indeed each a middle four interchange.

Therefore by Lemma 2.14 the braids $b_{n^{ \pm}}$obey $L^{\prime} b_{n^{ \pm}}=R^{\prime} b_{n^{ \pm}}$. Since the braids $b_{n^{ \pm}}$ are equal to their own 180 degree rotations, as mentioned in the introduction, this also implies that $L b_{n^{ \pm}}=R b_{n^{ \pm}}$. The internal unit conditions are fairly easy to verify by inspection of the braids $b_{n^{ \pm}}$; deleting the first two or the last two strands leaves the identity. The external unit conditions are checked just as easily if we again do so using the 180 degree rotations of $b_{n^{ \pm}}$.

For the converse we assume that $b$ is interchanging and unital and therefore by Lemma 3.3 it underlies an interchange $\eta_{(b)}$ in a braided category $\mathcal{V}$ seen as a 2-fold monoidal category with $\otimes_{1}=\otimes_{2}=\otimes$. We focus on the two strand sub-braids of $b$ underlying $\eta_{(b)_{A I I B}}$ (the outer sub-braid) and $\eta_{(b)_{I A B I}}$ (the inner sub-braid). We will now show that a selection of these two underlying braids uniquely determines the braid $b$.

First assume that we have chosen a two-strand braid to underlie the inner sub-braid of $b$. Consider the internal associativity axiom but with $U=W=Z=I$. Now the top horizontal arrow of the diagram has as its underlying braid the three-strand identity braid. The left vertical side of the diagram has the underlying braid formed by placing the underlying braid of $\eta_{(b)_{I X Y I}}$ (the inner sub-braid) to the right of a single strand. The right vertical side has the underlying braid of $\eta_{(b)_{I, V \otimes X, Y, I}}$. This latter is just the choice we made for the inner two-strand sub-braid of $b$, with the first strand doubled. The bottom horizontal arrow has the underlying braid of $\eta_{(b)_{I V Y X}}$. This last three strand sub-braid of $b$ is thus determined by the assumption that the diagram commutes, the braided coherence theorem, and the operation of taking the inverse in the braid group $B_{3}$. Thus we have determined the three strand sub-braid of $b$ formed by deleting the first strand.

Next we assume that we have chosen a braid to underlie $\eta_{(b)_{A I I B}}$. Then we again use the internal associativity diagram, this time with $V=X=Y=I$ to similarly determine the underlying braid of $\eta_{(b)_{U I W Z}}$, that is, the three strand sub-braid of $b$ formed by deleting the second strand.

Finally we set $V=W=I$ in the internal associativity diagram. Now the top horizontal arrow has the underlying braid formed by placing the outer two-strand sub-braid of $b$ to the left of the two-strand identity braid. The left vertical side has the underlying braid formed by placing a predetermined three strand sub-braid of $b$ (formed by deleting the first strand) to the right of a single strand. The bottom horizontal arrow has the underlying braid formed by doubling the last strand of a predetermined three-strand sub-braid of $b$ (formed by deleting the second strand). Thus by the operation of taking the inverse in the braid group $B_{4}$ we can determine the braid underlying the right vertical side. This is precisely the braid $b$, underlying $\eta_{(b)_{U X Y Z}}$. 
Next we will limit the choices we can make for the underlying braids of $\eta_{(b)_{I A B I}}$ and $\eta_{(b)_{A I I B}}$. We utilize Joyal and Street's result that, for any interchange $\eta$, a braiding is given by:

$$
A \otimes_{1} B \stackrel{\eta_{I A B I}}{\longrightarrow} B \otimes_{2} A \stackrel{\eta_{B I I A}^{-1}}{\longrightarrow} B \otimes_{1} A .
$$

Now our $\eta_{(b)_{I A B I}}$ and $\eta_{(b)_{A I I B}}$ have underlying two-strand braids. Thus by Lemma 2.6 and braided coherence we have the equation

$$
\eta_{(b)_{B I I A}}^{-1} \circ \eta_{(b)_{I A B I}}=c_{A B}^{ \pm 1} \quad \text { or } \quad \eta_{(b)_{I A B I}}=\eta_{(b)_{B I I A}} \circ c_{A B}^{ \pm 1} .
$$

Now in order for the permutation associated to $b$ to be $(23), \eta_{(b)_{I A B I}}$ must be an odd power of $c$ or $c^{-1}$. Therefore our choice for the underlying braids of $\eta_{(b)_{I A B I}}$ and $\eta_{(b)_{A I I B}}$ is reduced respectively to a choice of an odd integer $z$ and a choice of one of its neighboring integers $z \pm 1$. The choice of $z$ is the power of the $c$, and thus the power of $\sigma_{1}$ for the inner sub-braid. The latter choice of \pm 1 is the choice of the exponent of $c$ in the above equation, and thus determines the power of $\sigma_{1}$ for the outer sub-braid.

Now by Lemma 3.4 these possible choices for the underlying braids of $\eta_{(b)_{A I I B}}$ and $\eta_{(b)_{I A B I}}$ are all actually represented by one of the $b_{n^{ \pm}}$.

Therefore if any braid $b$ is interchanging and unital then it is equivalent to one of the braids $b_{n^{ \pm}}$.

The next item on the agenda is to investigate the equivalence of the various 2 -fold monoidal structures which can be constructed from a braiding, with differing underlying interchanging braids.

\section{Equivalence of 2-fold monoidal categories}

By finding interchanges which are formed from a braiding we have actually defined a collection of functors $F_{b}$ from the category of braided categories to the category of 2 -fold monoidal categories. The complete classification of interchanging unital braids is a well defined parametrization of this family.

4.1 Definition For $b$ an interchanging unital braid, the functor $F_{b}$ takes each braided category $\mathcal{V}$ to itself, seen as a 2 -fold monoidal category with interchange $\eta_{(b)}$. A braided tensor functor $f$ with $\phi: f(A) \otimes f(B) \rightarrow f(A \otimes B)$ is taken by $F_{b}$ to a 2-fold monoidal functor $F_{b}(f)$ which has the same definition on objects and morphisms and for which $\lambda^{1}=\lambda^{2}=\phi$. 
4.2 Theorem Given an interchanging unital braid $b$ the functor $F_{b}$ is naturally equivalent to either $F_{b_{0^{+}}}$or to $F_{b_{0^{-}}}$but not to both.

Proof It is directly implied by Joyal and Street [9] that given a 2-fold monoidal category $\mathcal{V}$ with $\otimes_{1}=\otimes_{2}$ and with strong interchange $\eta$ then that category is equivalent to the 2 -fold monoidal category $\mathcal{V}^{\prime}$ with the same objects and morphisms but with interchange given by

$$
\eta_{A B C D}^{\prime}=1_{A} \otimes\left(\eta_{C I I B}^{-1} \circ \eta_{I B C I}\right) \otimes 1_{D}
$$

For $\mathcal{V}$ braided and in terms of an original interchange $\eta_{(b)}$ based on a braiding $c$ with $b$ interchanging and unital, we have seen in the proof of Theorem 3.6 that $\eta_{A B C D}^{\prime}=$ $1_{A} \otimes c_{B C}^{ \pm 1} \otimes 1_{D}$. Thus $\mathcal{V}^{\prime}=F_{b_{0^{ \pm}}}(\mathcal{V})$. The 2 -fold monoidal functorial equivalence $U_{\mathcal{V}}: F_{b_{0^{ \pm}}}(\mathcal{V}) \rightarrow F_{b}(\mathcal{V})$ is the identity on objects and morphisms. Explicitly $U_{\mathcal{V}}$ has $\lambda_{A B}^{2}=1_{A \otimes B}$ and $\lambda_{A B}^{1}=\eta_{(b)_{A I I B}}$. This allows us to define in the target category:

$$
\eta_{U_{\mathcal{V}}(A) U_{\mathcal{V}}(B) U_{\mathcal{V}}(C) U_{\mathcal{V}}(D)}=\eta_{(b)_{A B C D}}
$$

The required hexagonal interchange diagram commutes due to braided coherence, using the braid equalities mentioned in Remark 2.16.

For $b$ such that $\eta_{(b)_{C I I B}}^{-1} \circ \eta_{(b)_{I B C I}}=c$, that is,

$$
b \in\left\{b_{n^{+}} \mid n \text { is even }\right\} \cup\left\{b_{n^{-}} \mid n \text { is odd }\right\},
$$

the family of functors $U_{\mathcal{V}}$ make up a natural isomorphism $U: F_{b_{0^{+}}} \rightarrow F_{b}$.

For $b$ such that $\eta_{(b)_{C I I B}}^{-1} \circ \eta_{(b)_{I B C I}}=c^{-1}$, that is,

$$
b \in\left\{b_{n^{+}} \mid n \text { is odd }\right\} \cup\left\{b_{n^{-}} \mid n \text { is even }\right\},
$$

the family of functors $U_{\mathcal{V}}$ make up a natural isomorphism $U: F_{b_{0^{-}}} \rightarrow F_{b}$.

There is not in general a natural isomorphism from $F_{b_{0}-}$ to $F_{b_{0^{+}}}$. If there were then the hexagonal interchange diagram for 2-fold monoidal functors with $A=D=I$ would become the diagram of braided equivalence between $\mathcal{V}$ with braiding $c$ and $\mathcal{V}$ with braiding $c^{-1}$. There is not in general a braided equivalence between $\mathcal{V}$ with braiding $c$ and $\mathcal{V}$ with braiding $c^{-1}$ since any $\lambda^{2}$ (in general based upon $c$ ) would have to satisfy $\lambda^{2} \circ c^{-1}=c \circ \lambda^{2}$ which is precluded by the braided coherence theorem and the fact that $B_{2}$ is abelian.

Thus the interchanging braids can be divided into two equivalence classes by the relation given by $b \equiv b^{\prime}$ if $F_{b}$ is equivalent to $F_{b}^{\prime}$. The two classes are canonically represented by the braids $b_{0^{+}}$and $b_{0^{-}}$. It would be an interesting future study to consider the 
braid groups $B_{n}$ for $n \geq 4$ modulo that equivalence relation on the first four strands. With that in mind we turn to examine some shortcuts to determining whether a given braid is interchanging and unital.

\section{Obstructions to being an interchange}

The general scheme is to find extra conditions on the interchange $\eta_{(b)}$ which together with the unit conditions and the associativity conditions will force the underlying braid $b$ to have easily checked characteristics. Then we can find families of unital braids in $B_{4}$ which cannot underlie an interchange, that is, which are not equivalent to any braid $b_{n^{ \pm}}$.

5.1 Theorem Given an interchange candidate braid $b$ with the property that deleting either the 2nd or 3rd strand gives the identity braid on three strands, then $b$ is interchanging if and only if $b=\sigma_{2}$, the second generator of $B_{4}$, or its inverse.

Proof This follows the logic of Balteanu, Fiedorowicz, Schwänzl and Vogt [3]. Letting $\eta=\eta_{(b)}$ be the interchange based on the braiding of $\mathcal{V}$ with underlying braid $b$, note that deleting a strand in $b$ corresponds to replacing the respective object in the product $A \otimes B \otimes C \otimes D$ with the identity $I$. Now let $V=W=I$ in the internal associativity diagram to see that due to the hypotheses on $b$ we have that $\eta_{U X Y Z}=1_{U} \otimes \eta_{I X Y Z}$. Then let $X=Y=I$ in the internal associativity diagram to see that $\eta_{U V W Z}=$ $\eta_{U V W I} \otimes 1_{Z}$. Together these two facts imply that $\eta_{A B C D}=1_{A} \otimes \eta_{I B C I} \otimes 1_{D}$. Then if we take $U=Z=W=0$ in the internal associativity law we get the first axiom of a braided category for $c_{B C}^{\prime}=\eta_{I B C I}$, and letting $U=Z=X=0$ in the internal associativity diagram gives the other one. This then implies that either $c^{\prime}=c$ or $c^{\prime}=c^{-1}$, since no other combinations of $c$ give a braiding. Therefore $\eta_{A B C D}=1_{A} \otimes c_{B C}^{ \pm 1} \otimes 1_{D}$ which has the underlying braid $\sigma_{2}^{ \pm 1}$. The converse is also clear from this discussion, since all the implications can be reversed. Of course, we already have the converse since the braids $b_{0^{ \pm}}$are interchanging.

This sort of obstruction can rule out candidate braids such as the braid $b_{(4)}$ in Example 2.9. It also rules out all but one element each of the left and right $\sigma_{2}^{ \pm(2 n-1)}$-cosets of the Brunnian braids in $B_{4}$, where the Brunnian braids are those pure braids where any strand deletion gives the identity braid. Even more broadly this obstruction rules out 
braids such as

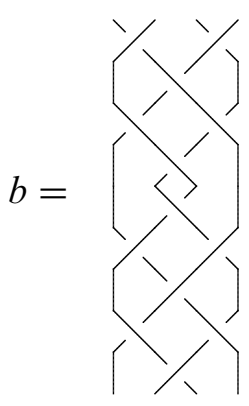

5.2 Theorem Let $b$ be an interchange candidate braid with the property that deleting both the inner two strands leaves the identity sub-braid on the remaining two strands. Then if $b$ is interchanging it follows that deleting either the second or the third strand will result in the three strand identity sub-braid on either of the remaining subsets of strands.

Proof Let $\eta_{(b)}$ be the interchange based on the braiding, with underlying interchanging braid $b$. We are given that $\eta_{(b)_{A I I B}}=1_{A \otimes B}$ and must demonstrate that $\eta_{(b)_{A I B C}}=$ $\eta_{(b)_{A B I C}}=1_{A \otimes B \otimes C}$. The conclusion about the deletion of the second strand is shown by considering the internal associativity diagram with $V=X=Y=I$. The conclusion about the deletion of the third strand is shown by considering the internal associativity diagram with $V=W=Y=I$. An alternative proof just uses the main results to check all the interchanging braids which fit the hypothesis.

This obstruction rules out all but one element each of the left and right $\sigma_{2}^{ \pm(2 n-1)}$ cosets of the 2-trivial or 2-decomposable braids in $B_{4}$. These latter braids are a generalization of the Brunnian braids in which deletion of any 2 strands results in a trivial braid.

Notice that the longer interchanging braids $b_{n^{ \pm}}$for $n>0$ give examples of interchanges that do not fit the conditions of the obstruction theorems so far. They also serve as examples of interchanges $\eta$ such that $\eta_{I B C I}$ is not a braiding. Recall however that they do give a braiding via $c_{A B}^{\prime}=\eta_{B I I A}^{-1} \circ \eta_{I A B I}$ as predicted by Joyal and Street. The latter condition also serves as a source of obstructions on its own. According to their theorem, any interchanging braid will have the property that dropping the outer two strands will give a two strand braid with one more or one less crossing of the same handedness than the two strand braid achieved by dropping the inner two strands. Indeed this condition rules out some of the same braids just mentioned, namely the Brunnian cosets of higher powers of $\sigma_{2}$ in $B_{4}$. 
The next sort of obstruction is found by slightly weakening the extra conditions. This will allow us to rule out a larger, different class of candidates, but they will be a little bit harder to recognize.

5.3 Theorem Let $b$ be an interchange candidate braid with the property that deleting either the first or the fourth strand results in a 3-strand braid that is just a power of the braid generator on what were the middle two strands: $\sigma_{i}^{ \pm n} ; i=2$ or $i=1$ respective of whether the first or fourth strand was deleted. Then $b$ is interchanging implies that $n=1$.

Proof The strand deletion conditions on the underlying braid $b$ of $\eta$ are equivalent to assuming that $\eta_{I B C D}=\eta_{I B C I} \otimes 1_{D}$ and that $\eta_{A B C I}=1_{A} \otimes \eta_{I B C I}$. Of course the power of the generator $\sigma_{i}$ being \pm 1 is equivalent to saying that $\eta_{I B C I}$ is the braiding $c$ or its inverse. Hence we need only show that the assumptions imply that $\eta_{I B C I}$ is a braiding. This is seen immediately upon letting $U=W=Z=I$ in the internal associativity axiom to get the first axiom of a braiding and letting $U=X=Z=I$ to get the other one.

This theorem can directly rule out candidates which satisfy the Joyal and Street condition that $c_{A B}=\eta_{B I I A}^{-1} \circ \eta_{I A B I}$ and the first or last strand deletion condition given here, but which fail to give a single crossing braid upon that removal. The simplest example is this braid:

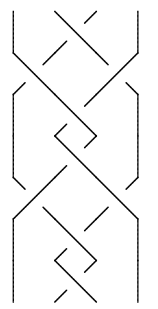

It is also true that a candidate braid which yields a single crossing after deletion of the first and fourth strands, if interchanging, must then obey the condition that deleting the first or last strand frees the other of those two from any crossings. This can be most easily seen by use of the main result; we simply check all four examples of interchanging unital braids which have inner two strand sub-braids a single crossing. They are $b_{0^{ \pm}}$and $b_{1^{ \pm}}$.

\section{Obstructions to braiding in $\mathcal{V}$-Cat}

Notice that in the case of symmetric $\mathcal{V}$ the axioms of enriched categories for $\mathcal{A} \otimes \mathcal{B}$ and the existence of a coherent 2-natural associator follow from the coherence of 
symmetric categories and the enriched axioms for $\mathcal{A}$ and $\mathcal{B}$. It remains to consider just why it is that $\mathcal{V}$-Cat is braided if and only if $\mathcal{V}$ is symmetric, and that if so then $\mathcal{V}$-Cat is symmetric as well. This fact is stated by Joyal and Street [9]. We choose to give a proof here which covers all possible interchanging braids explicitly, and all potential braidings on $\mathcal{V}$-Cat based on any odd power of the braiding on $\mathcal{V}$, by appealing to information from the theory of knots and links. This is opposed to arguments based on the fact that a braiding transports over a tensor equivalence, and on Theorem 4.2.

Our choice allows us to demonstrate how low dimensional topology can inform category theory as well as vice versa. A braiding $c^{(1)}$ on $\mathcal{V}$-Cat is a 2-natural transformation so $c_{\mathcal{A B}}^{(1)}$ is a $\mathcal{V}$-functor $\mathcal{A} \otimes \mathcal{B} \rightarrow \mathcal{B} \otimes \mathcal{A}$. On objects $c_{\mathcal{A B}}^{(1)}((A, B))=(B, A)$. Now to be precise we define $c^{(1)}$ to be based upon $c$ to mean that

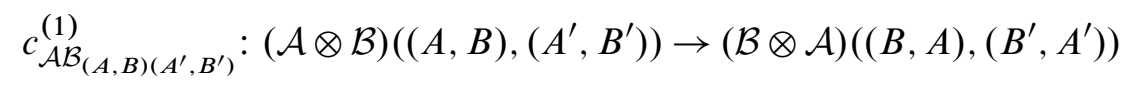

is defined to be

$$
c_{\mathcal{A}\left(A, A^{\prime}\right) \mathcal{B}\left(B, B^{\prime}\right)}: \mathcal{A}\left(A, A^{\prime}\right) \otimes \mathcal{B}\left(B, B^{\prime}\right) \rightarrow \mathcal{B}\left(B, B^{\prime}\right) \otimes \mathcal{A}\left(A, A^{\prime}\right) .
$$

This potential braiding must be checked for $\mathcal{V}$-functoriality. Again the unit axioms are trivial and we consider the more interesting associativity of hom-object morphisms property. The following diagram must commute:

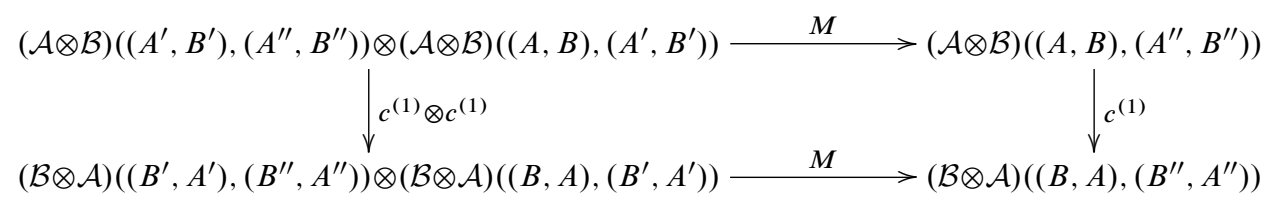

Let $X=\mathcal{A}\left(A^{\prime}, A^{\prime \prime}\right), Y=\mathcal{B}\left(B^{\prime}, B^{\prime \prime}\right), Z=\mathcal{A}\left(A, A^{\prime}\right)$ and $W=\mathcal{B}\left(B, B^{\prime}\right)$. Then expanding the above diagram using the composition defined as above (denoting various 
composites of $\alpha$ by unlabeled arrows) we have

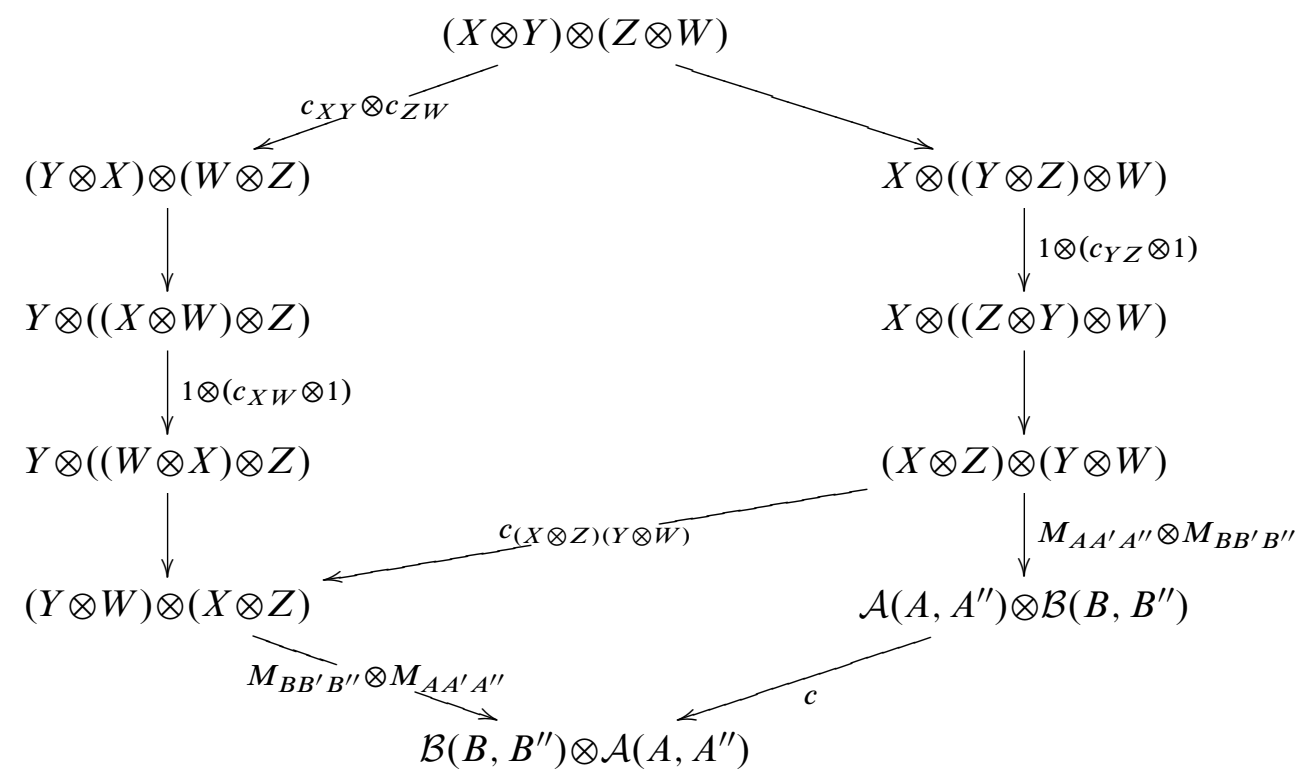

The bottom quadrilateral commutes by naturality of $c$. The top region must then commute for the diagram to commute, but the left and right legs have the following underlying braids

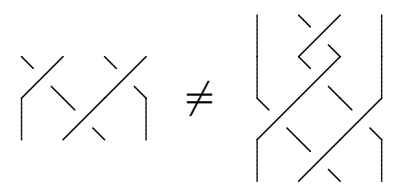

Thus as noted by Joyal and Street [9] neither braid $b_{(1)}$ nor its inverse can in general give a monoidal structure with a braiding based on the original braiding. In fact, it is easy to show more.

6.1 Theorem Let $\mathcal{V}$ be a braided category with braiding $c$. Let the tensor product on $\mathcal{V}-$ Cat be given by the canonical choices for the objects, hom-objects, unit morphisms, unit enriched category, and associator, and let $b$ be the underlying braid of the composition morphisms for the tensor product of enriched categories. Then in general there will not be a braiding in $\mathcal{V}$-Cat based upon the braiding $c$ in $\mathcal{V}$. Moreover, this failure will also be the case for attempts to produce a braiding in $\mathcal{V}$-Cat based upon any (odd) power $c^{2 n+1}$.

Proof Notice that in the above braid inequality each side of the inequality consists of the braid which underlies the definition of the composition morphism, in this case $b_{(1)}$, and an additional braid which underlies the segment of the preceding diagram 
that corresponds to a composite of $c^{(1)}$. In terms of braid generators the left side of the braid inequality begins with $\sigma_{1} \sigma_{3}$ corresponding to $c_{X Y} \otimes c_{Z W}$ and the right side of the braid inequality ends with $\sigma_{2} \sigma_{1} \sigma_{3} \sigma_{2}$ corresponding to $c_{(X \otimes Z)(Y \otimes W)}$. Since the same braid $b$ must end the left side as begins the right side, then for the diagram to commute we require $b \sigma_{1} \sigma_{3}=\sigma_{2} \sigma_{1} \sigma_{3} \sigma_{2} b$. This implies $\sigma_{1} \sigma_{3}=b^{-1} \sigma_{2} \sigma_{1} \sigma_{3} \sigma_{2} b$, or that the braids $\sigma_{1} \sigma_{3}$ and $\sigma_{2} \sigma_{1} \sigma_{3} \sigma_{2}$ are conjugate in $B_{4}$. Conjugate braids have precisely the same link as their closures, but the closure of $\sigma_{1} \sigma_{3}$ is an unlinked pair of circles whereas the closure of $\sigma_{2} \sigma_{1} \sigma_{3} \sigma_{2}$ is the Hopf link.
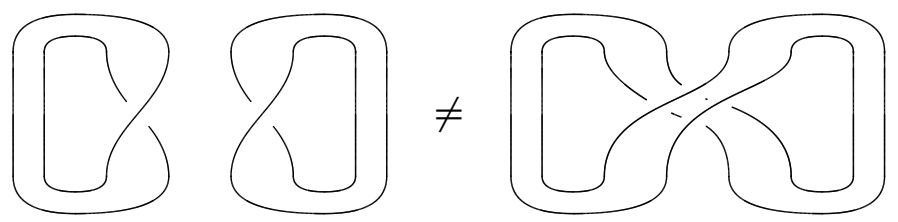

If we instead let

$$
c_{\mathcal{A} \mathcal{B}_{(A, B)\left(A^{\prime}, B^{\prime}\right)}^{(1)}}^{(1)}=c_{\mathcal{A}\left(A, A^{\prime}\right) \mathcal{B}\left(B, B^{\prime}\right)}^{2 n+1}
$$

then the requirement becomes that the braids $\left(\sigma_{1} \sigma_{3}\right)^{2 n+1}$ and $\left(\sigma_{2} \sigma_{1} \sigma_{3} \sigma_{2}\right)^{2 n+1}$ are conjugate in $B_{4}$. Both braids have as closure a link of two components-two copies of the $(2 n+1,2)$-torus knot. However the first closure is two unlinked copies of the knot while in the second closure the two (cabled) copies are linked with linking number $2 n+1$. Thus the braids cannot be conjugate, and so the braids underlying the legs of the functoriality diagram will not be equal for any choice of middle four interchange.

6.2 Corollary It is also interesting to note that the braid inequality above is the 180 degree rotation of the one which implies that in general $(\mathcal{A} \otimes \mathcal{B})^{\mathrm{op}} \neq \mathcal{A}^{\mathrm{op}} \otimes \mathcal{B}^{\mathrm{op}}$. Thus the proof also implies that the latter inequality holds in general for a tensor products of enriched categories with any braid $b$ underlying their composition morphisms, as well as any power of op as the exponent.

6.3 Remark It is quickly seen that if $c$ is a symmetry then in the second half of the braid inequality the upper portion of the braid consists of $c_{Y Z}$ and $c_{Z Y}=c_{Y Z}^{-1}$ so in fact equality holds. In that case then the derived braiding $c^{(1)}$ is a symmetry simply due to the definition.

\section{Implications for operads}

So far herein we have completely characterized families of interchanges based on a braiding which can define either a 2 -fold monoidal structure on a category or a 
monoidal structure on a 2-category. Another common use of a braiding is to define a monoidal structure on a category of collections, as in the theory of operads. Operads in a 2-fold monoidal category are defined as monoids in a certain category of collections by Forcey, Siehler and Sowers [7]. Here we repeat the basic ideas and the expanded definition in terms of commuting diagrams. The two principle components of an operad are a collection, historically a sequence, of objects in a monoidal category and a family of composition maps. Operads are often described as parameterizations of $n$-ary operations. Peter May's original definition of operad in a symmetric (or braided) monoidal category [12] has a composition $\gamma$ that takes the tensor product of the $n^{\text {th }}$ object ( $n$-ary operation) and $n$ others (of various arity) to a resultant that sums the arities of those others. The $n^{\text {th }}$ object or $n$-ary operation is often pictured as a tree with $n$ leaves, and the composition appears like this:
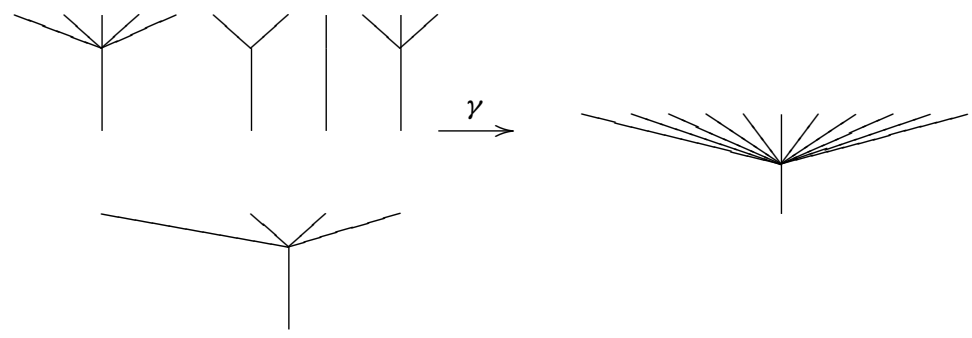

By requiring this composition to be associative we mean that it obeys this sort of pictured commuting diagram:

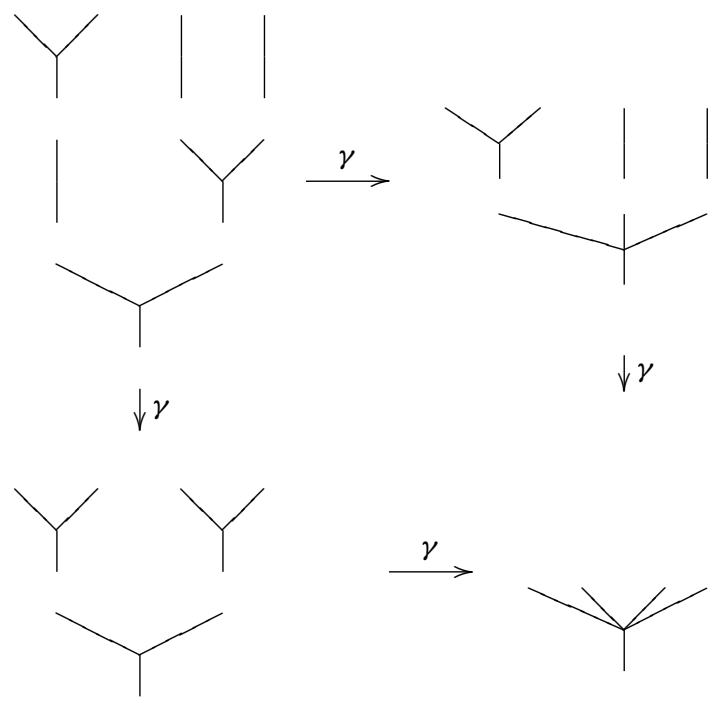

Algebraic $8 \mathcal{G}$ Geometric Topology, Volume 7 (2007) 
In the above pictures the tensor products are shown just by juxtaposition, but now we would like to think about the products more explicitly. If the monoidal category is not strict, then there is actually required another leg of the associativity diagram, where the tensoring is reconfigured so that the composition can operate in an alternate order. Here is how that rearranging looks in a symmetric (braided) category, where the shuffling is accomplished by use of the symmetry (braiding):

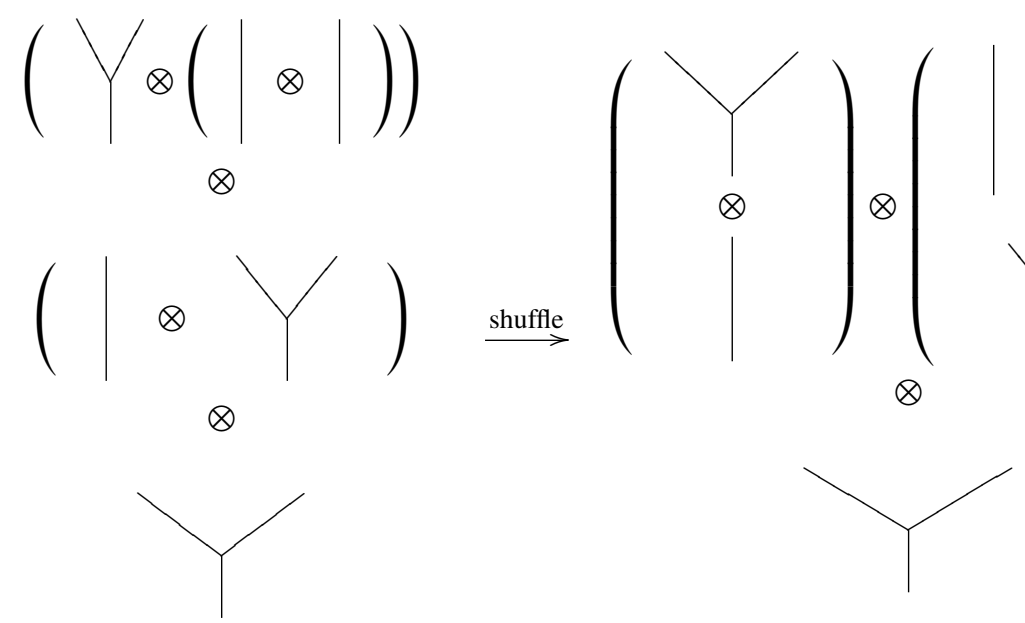

We now foreshadow our definition of operads in an iterated monoidal category with the same picture as above but using two tensor products, $\otimes_{1}$ and $\otimes_{2}$. It becomes clear that the true nature of the shuffle is in fact that of an interchange transformation.

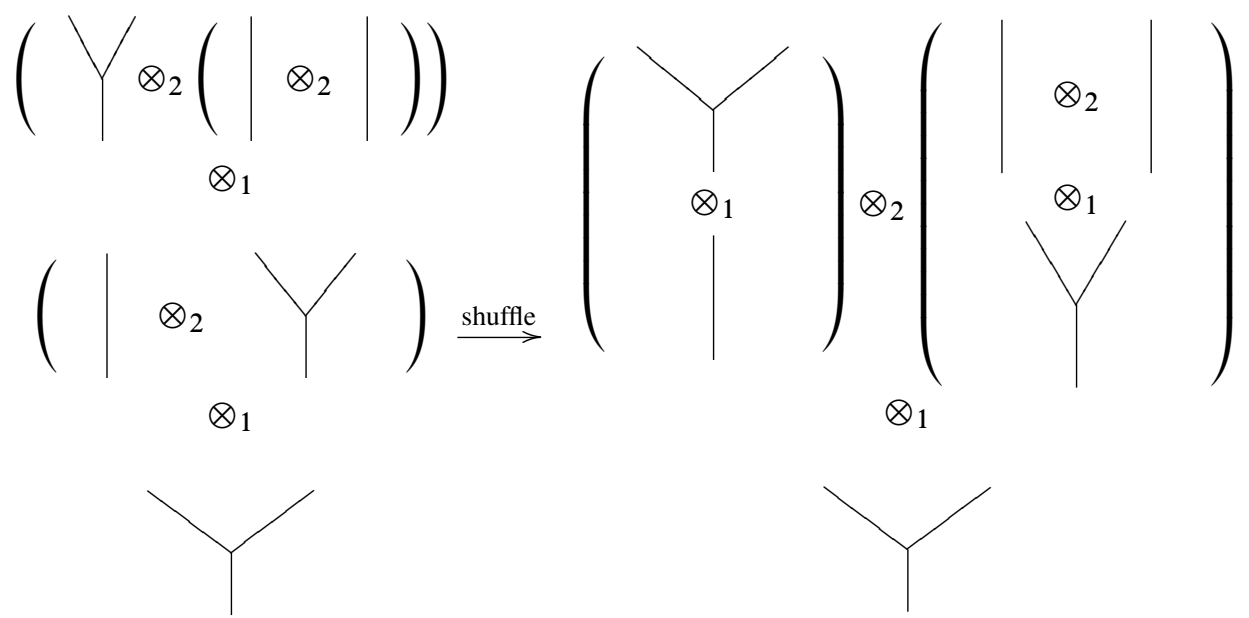

To see this just focus on the actual domain and range of $\eta$ which are the upper two levels of trees in the pictures, with the tensor product $\left(\left|\otimes_{2}\right|\right)$ considered as a single object. 
Now we are ready to give the technical definitions. We begin with the definition of 2 -fold operad in an $n$-fold monoidal category, as in the above picture, and then mention how it generalizes the case of operad in a braided category.

7.1 Definition Let $\mathcal{V}$ be a strict 2-fold monoidal category. A 2-fold operad $\mathcal{C}$ in $\mathcal{V}$ consists of objects $\mathcal{C}(j), j \geq 0$, a unit map $\mathcal{J}: I \rightarrow \mathcal{C}(1)$, and composition maps in $\mathcal{V}$

$$
\gamma^{12}: \mathcal{C}(k) \otimes_{1}\left(\mathcal{C}\left(j_{1}\right) \otimes_{2} \cdots \otimes_{2} \mathcal{C}\left(j_{k}\right)\right) \rightarrow \mathcal{C}(j)
$$

for $k \geq 1, j_{s} \geq 0$ for $s=1 \ldots k$ and $\sum_{n=1}^{k} j_{n}=j$. The composition maps obey the following axioms:

(1) Associativity: The following diagram is required to commute for all $k \geq 1, j_{s} \geq 0$ and $i_{t} \geq 0$, and where $\sum_{s=1}^{k} j_{s}=j$ and $\sum_{t=1}^{j} i_{t}=i$. Let $g_{s}=\sum_{u=1}^{s} j_{u}$ and let $h_{s}=\sum_{u=1+g_{s-1}}^{g_{s}} i_{u}$. The $\eta$ labeling the leftmost arrow actually stands for a variety of equivalent maps which factor into instances of the interchange.

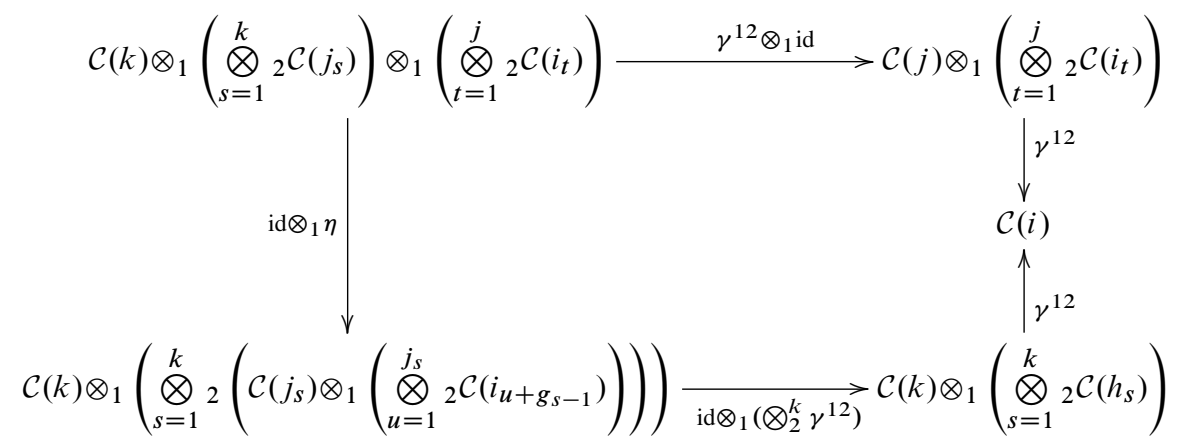

(2) Respect of units is required just as in the symmetric case. The following unit diagrams commute.
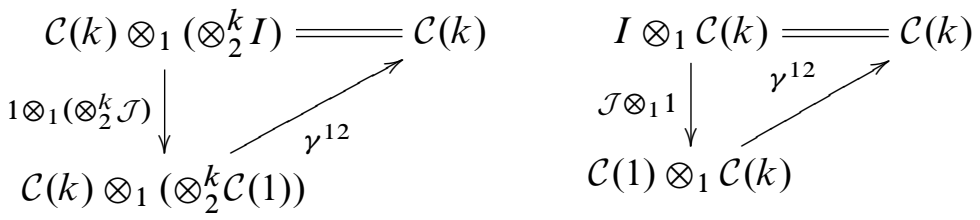

Now the problem of describing the various sorts of operads in a braided monoidal category becomes more clear, as a special case. Here again we let $\otimes=\otimes_{1}=\otimes_{2}$. The family of 2-fold structures based on interchanging braids gives rise to a family of monoidal structures on the category of collections, and thus to a family of operad structures. 
In the operad picture the underlying braid of an operad structure only becomes important when we inspect the various ways of composing a product with 4 levels of trees in the heuristic diagram, such as $\mathcal{C}(2) \otimes(\mathcal{C}(1) \otimes \mathcal{C}(1)) \otimes(\mathcal{C}(1) \otimes \mathcal{C}(1)) \otimes(\mathcal{C}(1) \otimes \mathcal{C}(1))$. For this composition to be well defined we require the internal associativity of the interchange that is used to rearrange the terms. When we consider composing a product with 3 levels of trees in the heuristic diagram, but with a base term $\mathcal{C}(n)$ with $n \geq 3$, such as: $\mathcal{C}(3) \otimes(\mathcal{C}(1) \otimes \mathcal{C}(1) \otimes \mathcal{C}(1)) \otimes(\mathcal{C}(1) \otimes \mathcal{C}(1) \otimes \mathcal{C}(1))$, then we see that the external associativity of $\eta$ is also required.

Thus the same theorems proven above for interchanging and non-interchanging families of braids apply here as well, in deciding whether a certain braid based shuffling of the terms in an operad product is allowable. The point is that not all shuffles using a braiding make sense, and the viewpoint of the 2-fold monoidal structure is precisely what is needed to see which shuffles do make sense. By seeing various shuffles as being interchanges on a fourfold product rather than braidings on a simple binary product, we are able to describe an infinite family of distinct compositions of the braiding each leading to well defined operad structure. The underlying braids are precisely those we denoted $b_{n^{ \pm}}$. In summary, structures based on a braiding are at worst ill-defined, at best defined up to equivalence, unless a 2-fold monoidal structure is chosen. Often in the literature the default is understood to be the simplest such structure where $\eta_{A B C D}=1_{A} \otimes c_{B C} \otimes 1_{D}$, but to be careful this choice should be made explicit. We have directly addressed operads and tensor products of enriched categories. The results herein should also be applied to $\mathcal{V}$-Act, the category of categories with an action of a monoidal category as described by McCrudden [13], as well as to $\mathcal{V}$-Mod, the bicategory of enriched categories and modules as described by Day and Street [4].

\section{References}

[1] J C Baez, Higher-dimensional algebra II: 2-Hilbert spaces, Adv. Math. 127 (1997) 125-189 MR1448713

[2] J C Baez, J Dolan, Categorification, from: "Higher category theory (Evanston, IL, 1997)", Contemp. Math. 230, Amer. Math. Soc., Providence, RI (1998) 1-36 MR1664990

[3] C Balteanu, Z Fiedorowicz, R Schwänzl, R Vogt, Iterated monoidal categories, Adv. Math. 176 (2003) 277-349 MR1982884

[4] B Day, R Street, Monoidal bicategories and Hopf algebroids, Adv. Math. 129 (1997) 99-157

[5] S Eilenberg, G M Kelly, Closed categories, from: "Proc. Conf. Categorical Algebra (La Jolla, CA, 1965)", Springer, New York (1966) 421-562 MR0225841 
[6] S Forcey, Enrichment over iterated monoidal categories, Algebr. Geom. Topol. 4 (2004) 95-119 MR2059184

[7] S Forcey, J Siehler, E S Sowers, Operads in iterated monoidal categories, J. Homotopy Relat. Struct. 2 (2007) 1-43

[8] F A Garside, The braid group and other groups, Quart. J. Math. Oxford Ser. (2) 20 (1969) 235-254 MR0248801

[9] A Joyal, R Street, Braided tensor categories, Adv. Math. 102 (1993) 20-78 MR1250465

[10] G M Kelly, Basic concepts of enriched category theory, London Mathematical Society Lecture Note Series 64, Cambridge University Press, Cambridge (1982) MR651714

[11] S Mac Lane, Categories for the working mathematician, second edition, Graduate Texts in Mathematics 5, Springer, New York (1998) MR1712872

[12] JP May, The geometry of iterated loop spaces, Lecture Notes in Mathematics 271, Springer, Berlin (1972) MR0420610

[13] P McCrudden, Balanced coalgebroids, Theory Appl. Categ. 7 (2000) No. 6, 71-147 MR1764504

[14] MC Shum, Tortile tensor categories, J. Pure Appl. Algebra 93 (1994) 57-110 MR1268782

Department of Physics and Mathematics, Tennessee State University

Nashville TN 37209, USA

Department of Mathematics, College of the Bahamas

PO Box N4912 Nassau, Bahamas

sforcey@tnstate.edu, felitahume@hotmail.com

http://faculty.tnstate.edu/sforcey/

Received: 17 January $2007 \quad$ Revised: 12 July 2007 